\title{
A comparison between two logical formalisms for rewriting
}

\author{
MIGUEL PALOMINO* \\ Departamento de Sistemas Informáticos y Programación \\ Facultad de Informática, Universidad Complutense de Madrid, Spain \\ (e-mail: miguelpt@sip.ucm.es) \\ submitted 12 December 2003; revised 12 April 2005; accepted 5 January 2006
}

\begin{abstract}
Meseguer's rewriting logic and the rewriting logic CRWL are two well-known approaches to rewriting as logical deduction that, despite some clear similarities, were designed with different objectives. Here we study the relationships between them, both at a syntactic and at a semantic level. Even though it is not possible to establish an entailment system map between them, both can be naturally simulated in each other. Semantically, there is no embedding between the corresponding institutions. Along the way, the notions of entailment and satisfaction in Meseguer's rewriting logic are generalized. We also use the syntactic results to prove reflective properties of CRWL.
\end{abstract}

KEYWORDS: rewriting logic, constructor-based rewriting logic, institutions

\section{Introduction}

The aim of this paper is to study in detail, and to try to clarify, the relationships between two well-known approaches to rewriting as logical deduction, namely, José Meseguer's rewriting logic (Meseguer 1992), and the constructor-based rewriting logic (CRWL) developed by Mario Rodríguez-Artalejo's research group in Madrid (González-Moreno et al. 1999).

The first of these was proposed as a logical framework wherein to represent other logics, and also as a semantic framework, a unified model of concurrency for the specification of languages and systems. The experience accumulated throughout the last years has come to support that original intention (Martí-Oliet and Meseguer 2002b). In particular, it has been shown that rewriting logic is a very flexible framework in which many other logics, including first-order logic, intuitionistic logic, linear logic, Horn logic with equality, as well as any other logic with a sequent calculus, can be represented (Meseguer 2000; Martí-Oliet and Meseguer 2002a: Verdejo and Martí-Oliet 2002 Thati et al. 2002 Verdejo 2003). An important feature of these representations that

\footnotetext{
* Supported by a postgraduate scholarship from the Spanish Ministry for Education, Culture, and Sports, and by the Spanish CICYT project AMEVA TIC 2000-0701-C02-01. This work was completed during a stay of the author at the Department of Computer Science in the University of Illinois at Urbana-Champaign.
} 
should be stressed is that they are usually quite simple and natural (in Meseguer's vocabulary, "the representation distance is zero"), so that the mathematical properties of the source logics are often straightforward to derive in their rewriting logic representation.

On the other hand, the goal of the constructor-based rewriting logic is to serve as a logical basis for declarative programming languages involving lazy evaluation, offering support, in addition, to non-strict and possibly non-deterministic functions.

Despite these differences, there is a clear resemblance between both logics, namely, the fact that logical deduction is based on rewriting. It seems natural, then, to ask about the relationships between deduction in these logics and to extend the question so as to encompass whether the corresponding models are also related. A suitable framework in which to carry out this study is the theory of general logics developed in Meseguer (1989). There, logics are described in a very abstract manner and two separated components are distinguished: a syntactic part, which is captured by the notion of entailment system, and a semantic one, captured by Goguen and Burstall's concept of institution (Goguen and Burstall 1992).

We will begin by studying derivability and, for that, we will try to associate entailment systems to both logics and to relate them by means of a map of entailment systems. Unfortunately, it will be proved that there is none corresponding to deduction in CRWL, so we will be forced to leave this formal framework and undertake more informal simulations of the logics in each other. Although such simulations are always possible by making use of suitable low-level encodings, relying on the analogies between both logics our interest resides in finding natural and simple simulations that at the very least would show that their expressive power is the same. In addition, these results will be used to study reflective properties of CRWL.

After the comparison at the syntactic level, the next step is the study of the corresponding models. Now we will be able to associate an institution to each logic, so this study will take place within the formal framework of the theory of institutions. The main result we will obtain is that models in these logics bear no relation at all. Along the way, we generalize the notions of derivability and satisfaction in Meseguer's rewriting logic to conditional rewrite rules, and clarify some subtle points regarding the definition of models in this logic.

As implied by the previous presentation, this paper does not consider the operational semantics of the logics, but focus instead of comparing them at a more abstract level by considering both its provability and satisfaction relations. We refer to works like Bosco et al. (1988), where such operational issues related to resolution or narrowing are pursued in similar contexts.

Meseguer's rewriting logic is parameterized with respect to an underlying equational logic; although this can be typed and as general as the membership equational logic from Meseguer (1998), in this paper we concentrate on the version of rewriting logic which uses unsorted and unconditional equational logic and write RL for it. Likewise, there are also typed versions of CRWL (Arenas-Sánchez and Rodríguez-Artalejo 2001 González-Moreno et al. 2001). Here we have chosen to focus on the untyped versions because this work is mainly foundational and the addition of types, while not introducing any new fundamental concepts, would force us to deal with many details that 
would obscure the presentation (for example, quantifiers would have to be considered explicitly). The typed cases are considered in some detail in Palomino (2001).

In what follows we assume familiarity with some basic ideas of category theory (Barr and Wells 1999), that do not go beyond natural transformations and equalizers. Only in Section 3.2 we use a less standard construction, that of subequalizer, that we describe in the text.

\section{Relations at the Syntactic Level}

In the first part of the paper we focus on the syntactic aspects of the logics, and try to abstractly study derivability in them at the level of entailment systems. After ruling out this possibility, we develop some simulations that will allow us to prove some reflective properties of the logics. We start by reviewing the main concepts and definitions that we will use.

\subsection{Entailment systems}

Syntax is typically given by a signature $\Sigma$ providing a grammar on which sentences, collected in a set $\operatorname{sen}(\Sigma)$, are built. For a given signature $\Sigma$, entailment (also called provability) of a sentence $\varphi \in \operatorname{sen}(\Sigma)$ from a set of axioms $\Gamma \subseteq \operatorname{sen}(\Sigma)$ is a relation $\Gamma \vdash \varphi$ which holds if and only if we can prove $\varphi$ from the axioms $\Gamma$ using the rules of the logic. We make this relation relative to a signature. In the rest of the paper, let $|\mathcal{C}|$ denote the collection of objects of a category $\mathcal{C}$.

An entailment system (Meseguer 1989) is a triple $\mathcal{E}=($ Sign, sen, $\vdash)$ such that:

- Sign is a category whose objects are called signatures.

- sen : Sign $\rightarrow$ Set is a functor associating to each signature $\Sigma$ a corresponding set of $\Sigma$-sentences.

- $\vdash$ is a function which associates to each $\Sigma \in|\mathbf{S i g n}|$ a binary relation $\vdash_{\Sigma} \subseteq$ $\mathcal{P}(\operatorname{sen}(\Sigma)) \times \operatorname{sen}(\Sigma)$ called $\Sigma$-entailment such that the following properties are satisfied:

1. reflexivity: for any $\varphi \in \operatorname{sen}(\Sigma),\{\varphi\} \vdash_{\Sigma} \varphi$,

2. monotonicity: if $\Gamma \vdash_{\Sigma} \varphi$ and $\Gamma^{\prime} \supseteq \Gamma$ then $\Gamma^{\prime} \vdash_{\Sigma} \varphi$,

3. transitivity: if $\Gamma \vdash_{\Sigma} \varphi_{i}$, for all $i \in I$, and $\Gamma \cup\left\{\varphi_{i} \mid i \in I\right\} \vdash_{\Sigma} \psi$, then $\Gamma \vdash_{\Sigma} \psi$

4. $\vdash$-translation: if $\Gamma \vdash_{\Sigma} \varphi$, then for any $H: \Sigma \rightarrow \Sigma^{\prime}$ in Sign, $\operatorname{sen}(H)(\Gamma) \vdash_{\Sigma^{\prime}}$ $\operatorname{sen}(H)(\varphi)$.

Given an entailment system $\mathcal{E}$, its category $\mathbf{T h}$ of theories has as objects pairs $T=(\Sigma, \Gamma)$, with $\Sigma$ a signature and $\Gamma \subseteq \operatorname{sen}(\Sigma)$. A theory morphism $H:(\Sigma, \Gamma) \rightarrow$ $\left(\Sigma^{\prime}, \Gamma^{\prime}\right)$ is a signature morphism $H: \Sigma \rightarrow \Sigma^{\prime}$ such that if $\varphi \in \Gamma$, then $\Gamma^{\prime} \vdash_{\Sigma^{\prime}}$ $\operatorname{sen}(H)(\varphi)$. A theory morphism is axiom-preserving if, in addition, it satisfies the condition $\operatorname{sen}(H)(\Gamma) \subseteq \Gamma^{\prime}$. This defines a subcategory $\mathbf{T h}_{0}$ with the same objects as $\mathbf{T h}$ but with morphisms restricted to be axiom-preserving theory morphisms, that does not depend on the entailment relation.

Although we will not be able to use it, there is also a notion of map of entailment 


$$
\begin{aligned}
& \overline{[t] \rightarrow[t]} \text { Reflexivity } \quad \frac{[t] \rightarrow\left[t^{\prime}\right] \quad\left[t^{\prime}\right] \rightarrow\left[t^{\prime \prime}\right]}{[t] \rightarrow\left[t^{\prime \prime}\right]} \text { Transitivity } \\
& \frac{\left[t_{1}\right] \rightarrow\left[t_{1}^{\prime}\right] \ldots\left[t_{n}\right] \rightarrow\left[t_{n}^{\prime}\right]}{\left[f\left(t_{1}, \ldots, t_{n}\right)\right] \rightarrow\left[f\left(t_{1}^{\prime}, \ldots, t_{n}^{\prime}\right)\right]} \text { Congruence } \\
& r:[t] \rightarrow\left[t^{\prime}\right] \text { if }\left[a_{1}\right] \rightarrow\left[b_{1}\right] \wedge \ldots \wedge\left[a_{m}\right] \rightarrow\left[b_{m}\right] \in \Gamma \\
& {\left[w_{1}\right] \rightarrow\left[w_{1}^{\prime}\right] \quad \ldots \quad\left[w_{n}\right] \rightarrow\left[w_{n}^{\prime}\right]} \\
& \frac{\left[a_{1}(\bar{w} / \bar{x})\right] \rightarrow\left[b_{1}(\bar{w} / \bar{x})\right] \quad \ldots\left[a_{m}(\bar{w} / \bar{x})\right] \rightarrow\left[b_{m}(\bar{w} / \bar{x})\right]}{[t(\bar{w} / \bar{x})] \rightarrow\left[t^{\prime}\left(\overline{w^{\prime}} / \bar{x}\right)\right]} \text { Replacement }
\end{aligned}
$$

Fig. 1. Rules of deduction for an RL-theory $(\Sigma, E, L, \Gamma)$

systems, allowing us to relate logics in a general and systematic way. Basically, a map of entailment systems $\mathcal{E} \rightarrow \mathcal{E}^{\prime}$ maps signatures of $\mathcal{E}$ to signatures of $\mathcal{E}^{\prime}$ (or, more generally, theories to theories), and sentences of $\mathcal{E}$ to sentences of $\mathcal{E}^{\prime}$, respecting the entailment relations $\vdash$ of $\mathcal{E}$ and $\vdash^{\prime}$ of $\mathcal{E}^{\prime}$. See Meseguer (1989) for details.

\subsection{Rewriting logic}

A signature in $\mathrm{RL}$ is a pair $(\Sigma, E)$ with $\Sigma$ a ranked alphabet of function symbols and $E$ a set of $\Sigma$-equations. Rewriting operates on equivalence classes of terms modulo the set of equations $E$. We denote by $T_{\Sigma}(\mathcal{X})$ the $\Sigma$-algebra of $\Sigma$-terms with variables in a set $\mathcal{X}$, and by $[t]_{E}$ or just $[t]$ the $E$-equivalence class of $t \in T_{\Sigma}(\mathcal{X})$. To indicate that $\left\{x_{1}, \ldots, x_{n}\right\}$ is the set of variables occurring in $t$ we write $t\left(x_{1}, \ldots, x_{n}\right)$. Given $t\left(x_{1}, \ldots, x_{n}\right)$, and terms $u_{1}, \ldots, u_{n}, t\left(u_{1} / x_{1}, \ldots, u_{n} / x_{n}\right)$ denotes the term obtained from $t$ by simultaneously substituting $u_{i}$ for $x_{i}, i=1, \ldots, n$. To simplify notation we denote a sequence of objects $a_{1}, \ldots, a_{n}$ by $\bar{a}$; with this notation, $t\left(u_{1} / x_{1}, \ldots, u_{n} / x_{n}\right)$ can be abbreviated to $t(\bar{u} / \bar{x})$.

An RL-theory $\mathcal{R}$ is a 4 -tuple $\mathcal{R}=(\Sigma, E, L, \Gamma)$, where $(\Sigma, E)$ is a signature and $\Gamma$ is a set of rewrite rules, labeled with elements of $L$, of the form

$$
r:[t] \rightarrow\left[t^{\prime}\right] \text { if }\left[a_{1}\right] \rightarrow\left[b_{1}\right] \wedge \ldots \wedge\left[a_{m}\right] \rightarrow\left[b_{m}\right] .
$$

We write $\mathcal{R} \vdash[t] \rightarrow\left[t^{\prime}\right]$ if $[t] \rightarrow\left[t^{\prime}\right]$ can be derived using the rules of deduction shown in Figure 1 for a complete exposition of RL we refer the reader to Meseguer (1992).

\section{$2.3 C R W L$}

CRWL uses signatures with constructors $\Sigma=C_{\Sigma} \cup F_{\Sigma}$, where $C_{\Sigma}=\bigcup_{n \in \mathbb{N}} C_{\Sigma}^{n}$ and $F_{\Sigma}=\bigcup_{n \in \mathbb{N}} F_{\Sigma}^{n}$ are disjoint sets of constructor and defined function symbols respectively, each of them with an associated arity. $\Sigma_{\perp}$ refers to the signature which is obtained from $\Sigma$ by adding a new constructor $\perp$ of arity 0 . Given a set $\mathcal{X}$ of variables, we will write $\operatorname{Expr}(\Sigma, \mathcal{X})$ for the set of total expressions which can be built with $\Sigma$ and $\mathcal{X}$, and $\operatorname{Term}(\Sigma, \mathcal{X})$ for those total terms which only make use of 


$$
\begin{aligned}
& \overline{e \rightarrow I} \text { Bottom } \quad \overline{e \rightarrow e} \text { Reflexivity } \\
& \frac{e_{1} \rightarrow e_{1}^{\prime} \ldots e_{n} \rightarrow e_{n}^{\prime}}{h\left(e_{1}, \ldots, e_{n}\right) \rightarrow h\left(e_{1}^{\prime}, \ldots, e_{n}^{\prime}\right)} \text { Monotonicity } \\
& \theta: \mathcal{X} \longrightarrow \operatorname{Term}_{\perp}(\Sigma, \mathcal{X}) \\
& l \rightarrow r \Leftarrow a_{1} \bowtie b_{1}, \ldots, a_{n} \bowtie b_{n} \in \Gamma \\
& \frac{\theta\left(a_{1}\right) \bowtie \theta\left(b_{1}\right) \ldots \theta\left(a_{n}\right) \bowtie \theta\left(b_{n}\right)}{\theta(l) \rightarrow \theta(r)} \text { Reduction } \\
& \frac{e \rightarrow e^{\prime} \quad e^{\prime} \rightarrow e^{\prime \prime}}{e \rightarrow e^{\prime \prime}} \text { Transitivity } \quad \frac{a \rightarrow t \quad b \rightarrow t \quad t \text { a total term }}{a \bowtie b} \text { Join }
\end{aligned}
$$

Fig. 2. Rules of deduction for a CRWL-theory $(\Sigma, \Gamma)$

$C_{\Sigma}$ and $\mathcal{X} . \operatorname{Expr}_{\perp}(\Sigma, \mathcal{X})$ and $\operatorname{Term}_{\perp}(\Sigma, \mathcal{X})$, the sets of partial expressions and terms, are defined analogously using $\Sigma_{\perp}$. A signature morphism (Molina-Bravo 2000) $\sigma$ : $\Sigma \rightarrow \Sigma^{\prime}$ from a signature $\Sigma=C_{\Sigma} \cup F_{\Sigma}$ to another $\Sigma^{\prime}=C_{\Sigma^{\prime}} \cup F_{\Sigma^{\prime}}$ is a pair of functions (denoted with the same $\sigma$ )

$$
\sigma: C_{\Sigma} \rightarrow C_{\Sigma^{\prime}} \text { and } \sigma: F_{\Sigma} \rightarrow F_{\Sigma^{\prime}}
$$

mapping $n$-ary symbols to $n$-ary symbols.

A CRWL-theory is a pair $(\Sigma, \Gamma)$, where $\Sigma$ is a signature with constructors and $\Gamma$ is a set of conditional rewrite rules of the form

$$
f\left(t_{1}, \ldots, t_{n}\right) \rightarrow r \Leftarrow a_{1} \bowtie b_{1}, \ldots, a_{m} \bowtie b_{m} \quad(m \geq 0),
$$

with $f \in F_{\Sigma}$ of arity $n, t_{1}, \ldots, t_{n} \in \operatorname{Term}(\Sigma, \mathcal{X}), r, a_{i}, b_{i} \in \operatorname{Expr}(\Sigma, \mathcal{X}), i=1, \ldots, m$, $r$ and each variable occurring in $t_{1}, \ldots, t_{n}$ having a single ocurrence.

From a given theory $T$, two kinds of sentences can be derived using the CRWLcalculus in Figure 2] (where variables range over partial expressions): reduction statements of the form $a \rightarrow b$, and joinability statements $a \bowtie b$ (meaning that there exists a total term to which both $a$ and $b$ reduce). We denote them by $T \vdash a \rightarrow b$ and $T \vdash a \bowtie b$, respectively. Again, we refer to González-Moreno et al. (1999) for a complete presentation of CRWL. (Note that the names "term" and "constructor term" are used there instead of "expression" and "term.")

\subsection{An entailment system for RL}

In order to associate an entailment system to RL, note that the rules of inference in Figure 1 only allow us to derive unconditional rules but that the requirements on an entailment system (reflexivity) require the ability to derive conditional ones as well. We then have two possibilities: either we restrict ourselves to unconditional rewrite rules and define $\vdash_{\Sigma}$ by means of derivation in the RL-calculus, or we also consider conditional rules, in which case the RL-calculus in Figure 1 must be extended to be able to derive them. We consider the second, more general case. 
Actually, not only is derivability undefined for conditional rules, but also is satisfaction. However, we would like to rest on a natural definition of satisfaction to support the claim that our extended notion of derivability is a suitable one. The semantics of RL is presented in Section 3.2 and the extension of the satisfaction relation discussed in Section 3.5 here we just assume that such an extension exists.

Given an RL-theory $\mathcal{R}=(\Sigma, E, L, \Gamma)$ and a set of variables $\mathcal{X}$ disjoint from $\Sigma$, we define $\mathcal{R}(\mathcal{X})=\left(\Sigma(\mathcal{X}), E, L, \Gamma^{\prime}\right)$ where $\Sigma(\mathcal{X})$ is the set of function symbols obtained by adding the elements of $\mathcal{X}$ as constants to $\Sigma$, and $\Gamma^{\prime}$ is obtained from $\Gamma$ by renaming with fresh variables. In Section 3.5 it is proved that, for an RL-theory $\mathcal{R}$ and $[t(\bar{x})] \rightarrow\left[t^{\prime}(\bar{x})\right]$ if $\left[a_{1}(\bar{x})\right] \rightarrow\left[b_{1}(\bar{x})\right] \wedge \ldots \wedge\left[a_{m}(\bar{x})\right] \rightarrow\left[b_{m}(\bar{x})\right]$ a conditional rewrite rule, the following statements are equivalent:

1. $\mathcal{R} \models[t(\bar{x})] \rightarrow\left[t^{\prime}(\bar{x})\right]$ if $\left[a_{1}(\bar{x})\right] \rightarrow\left[b_{1}(\bar{x})\right] \wedge \ldots \wedge\left[a_{m}(\bar{x})\right] \rightarrow\left[b_{m}(\bar{x})\right] ;$

2. $\mathcal{R}(\bar{x}) \cup\left\{\left[a_{1}(\bar{x})\right] \rightarrow\left[b_{1}(\bar{x})\right], \ldots,\left[a_{m}(\bar{x})\right] \rightarrow\left[b_{m}(\bar{x})\right]\right\} \models[t(\bar{x})] \rightarrow\left[t^{\prime}(\bar{x})\right]$.

A straightforward consequence of this equivalence is a sound and complete extension of the RL-calculus with the following rule of deduction:

- Implication introduction.

$$
\frac{\mathcal{R}(\bar{x}) \cup\left\{\left[a_{1}(\bar{x})\right] \rightarrow\left[b_{1}(\bar{x})\right], \ldots,\left[a_{m}(\bar{x})\right] \rightarrow\left[b_{m}(\bar{x})\right]\right\} \vdash[t(\bar{x})] \rightarrow\left[t^{\prime}(\bar{x})\right]}{\mathcal{R} \vdash[t(\bar{x})] \rightarrow\left[t^{\prime}(\bar{x})\right] \text { if }\left[a_{1}(\bar{x})\right] \rightarrow\left[b_{1}(\bar{x})\right] \wedge \ldots \wedge\left[a_{m}(\bar{x})\right] \rightarrow\left[b_{m}(\bar{x})\right]} .
$$

We can now focus again on the main purpose of this section. For that, we associate to RL the entailment sytem $\mathcal{E}_{\mathrm{RL}}=(\mathbf{S i g n}$, sen,,$)$ given by:

- Sign: the category of equational theories and theory morphisms;

- sen: the functor assigning to an equational theory the set of conditional rewrite rules that can be built over it, and mapping a theory morphism to its natural extension to rewrite rules;

- $\vdash$ is defined as provability in the extended RL-calculus.

Proposition 1

$\mathcal{E}_{\mathrm{RL}}=($ Sign, sen,$\vdash)$ is an entailment system.

The proof of this result uses concepts from the model theory of RL that are not introduced until later in the text, so we postpone the details to the appendix.

Before finishing, it should be emphasized that throughout this section no mention at all has been made of the labels in an RL-theory. They could have been safely included within the signature part; however, they do not play any role as far as the entailment relation is concerned and, if only for ease of exposition, we have preferred to omit then. This situation will change drastically when we shift to models and try to assign an institution to RL; then, we will be forced to distinguish between labeled and unlabeled sentences, as described in Section 3.5

\subsection{An entailment system for CRWL}

At first sight, an entailment system can be associated to CRWL following the same steps as for RL. The category of signatures is immediately obtained, as it is not 
difficult to check that composition of signature morphisms is associative, and for the set of sentences we have the same two possibilities as for RL.

However, a closer look reveals that derivation in the CRWL-calculus does not satisfy the transitivity condition for the provability relation in entailment systems. Consider, for example, a signature $\Sigma$ with $c, d, h \in \Sigma$, function symbols of arities 0,0 , and 1 , respectively. Then it can be proved that

$$
\{c \rightarrow h(c), h(x) \rightarrow h(d) \Leftarrow x \bowtie x\} \vdash_{\mathrm{CRWL}} h(x) \rightarrow h(d)
$$

and

$$
\{c \rightarrow h(c), h(x) \rightarrow h(d) \Leftarrow x \bowtie x, h(x) \rightarrow h(d)\} \vdash_{\mathrm{CRWL}} c \rightarrow h(d),
$$

but

$$
\{c \rightarrow h(c), h(x) \rightarrow h(d) \Leftarrow x \bowtie x\} \nvdash_{\mathrm{CRWL}} c \rightarrow h(d) .
$$

The first statement is proved by instantiating $h(x) \rightarrow h(d) \Leftarrow x \bowtie x$ with $x$; for the second, noting that $h(c) \rightarrow h(\perp)$ (using Bottom and Congruence), just instantiate $h(x) \rightarrow h(d)$ with $\perp$ and apply Transitivity (note that $c$ cannot be used to instantiate this rule since it is not a term). The third statement is formally proved by induction on derivations: let us just note that the crucial point is that the rule $h(x) \rightarrow h(d) \Leftarrow x \bowtie x$ cannot be instantiated with $\perp$ because $\perp \bowtie \perp$ cannot be derived. What lies behind is the fact that the CRWL-calculus is sound and complete with respect to validity in models only under totally defined valuations (González-Moreno et al. 1999). In particular, in the first entailment above, $h(x) \rightarrow$ $h(d)$ means that $h(t)$ rewrites to $h(d)$ just for those instances where a total term $t$ is substituted for $x$.

This proves that the relation $\vdash_{\mathrm{CRWL}}$ is not transitive and, therefore, we are not going to be able to build an entailment system based on the CRWL-calculus, as any sensible one should contain, at least, the conditional rewrite rules among its sentences. (Let us note, however, that there is an entailment system corresponding to the institution that will be associated to CRWL in Section 3.4 the previous example is no longer a counterexample due to the partiality of the soundness and completeness results for CRWL mentioned above. The reason for not comparing it to RL's entailment system is that, since it is not based on deduction, they do not stand "on the same ground.")

\subsection{Simulating CRWL in RL}

Since there is no entailment system corresponding to the CRWL-calculus, we cannot define a map of entailment systems as intended. In the following we will be pleased just with presenting how entailment in CRWL can be simulated in RL. The set of labels of an RL-theory does not take part in the entailment process, and so it is omitted; the same convention will also be adopted in Section 2.7

Of course, every CRWL-theory $T$ can be trivially "simulated" in RL by means of an RL-theory $T^{\prime}$ with a constant $c_{t}$ for each term (and each expression) $t$ in $T$, and with axioms $c_{t} \rightarrow c_{t^{\prime}}$ whenever $T \vdash t \rightarrow t^{\prime}$. But such a $T^{\prime}$, apart from not 
exploiting the analogies between RL and CRWL, is not computable in general. And so we must look for another construction.

The idea is to associate to every CRWL-theory $T=(\Sigma, \Gamma)$ a theory $T^{\prime}$ in RL (whose set of equational axioms will be empty) in which all the operations in $T$, together with a new constant $\perp$, are available, plus one rule for each axiom in $T$ and, perhaps, some more rules coping with the rules of deduction of the CRWL-calculus. Since rules in CRWL can only be instantiated with terms and not expressions and there is no such distinction in RL, we introduce a unary relation pterm (technically, a unary function symbol) and a constant true to distinguish them in RL. One immediate rule defining pterm is pterm $(\perp) \rightarrow$ true; however, how to express that variables are also partial terms? The obvious rule $\operatorname{pterm}(x) \rightarrow$ true is clearly not valid: everything would be a partial term! This means that we must consider the CRWL variables at the object level, add them to the signature of $T^{\prime}$ as constants, and use a new set $\mathcal{X}$ of variables for RL. Using constants for variables we will be able to distinguish those terms in RL representing terms in CRWL from those representing expressions, hence allowing us to capture, by carefully translating the rules of deduction of the CRWL-calculus (using, perhaps, a different representation for the terms appearing in them), the corresponding entailment relation.

Then, assuming variables in CRWL belong to a set $\mathcal{V}$, the rules defining pterm are:

$$
\begin{aligned}
& \operatorname{pterm}(\perp) \rightarrow \text { true } \\
& \operatorname{pterm}\left(v_{i}\right) \rightarrow \text { true } \quad\left(\forall v_{i} \in \mathcal{V}\right) \\
& \operatorname{pterm}\left(h\left(x_{1}, \ldots, x_{n}\right)\right) \rightarrow \operatorname{true} \\
& \quad \text { if } \operatorname{pterm}\left(x_{1}\right) \rightarrow \operatorname{true} \wedge \ldots \wedge \operatorname{pterm}\left(x_{n}\right) \rightarrow \operatorname{true} \quad\left(\forall h \in C_{\Sigma}^{n}, n \in \mathbb{N}\right)
\end{aligned}
$$

In a similar way, two more predicates, tterm and pexpr, dealing with total terms and partial expressions, are defined:

$$
\begin{aligned}
& \operatorname{tterm}\left(v_{i}\right) \rightarrow \text { true }\left(\forall v_{i} \in \mathcal{V}\right) \\
& \operatorname{tterm}\left(h\left(x_{1}, \ldots, x_{n}\right)\right) \rightarrow \text { true } \\
& \quad \text { if } \operatorname{tterm}\left(x_{1}\right) \rightarrow \operatorname{true} \wedge \ldots \wedge \operatorname{term}\left(x_{n}\right) \rightarrow \text { true } \quad\left(\forall h \in C_{\Sigma}^{n}, n \in \mathbb{N}\right) \\
& \operatorname{pexpr}(\perp) \rightarrow \text { true } \quad\left(\forall v_{i} \in \mathcal{V}\right) \\
& \operatorname{pexpr}\left(v_{i}\right) \rightarrow \text { true } \\
& \operatorname{pexpr}\left(h\left(x_{1}, \ldots, x_{n}\right)\right) \rightarrow \text { true } \\
& \quad \text { if } \operatorname{pexpr}\left(x_{1}\right) \rightarrow \operatorname{true} \wedge \ldots \wedge \operatorname{pexpr}\left(x_{n}\right) \rightarrow \text { true } \quad\left(\forall h \in C_{\Sigma}^{n} \cup F_{\Sigma}^{n}, n \in \mathbb{N}\right)
\end{aligned}
$$

As a side effect, rewriting in CRWL can no longer be simulated in RL directly through the rewriting relation. Consider, for example, the theory of natural numbers in CRWL, with 0 a constructor and + a function symbol. In RL, pterm $(0+0)$ should not rewrite to true; however, with the usual definitions, $0+0 \rightarrow 0$ and by Congruence pterm $(0+0) \rightarrow$ pterm $(0)$, and this last term must reduce to true. Therefore, a rewrite in CRWL will be simulated through a binary relation $R$ so that $e \rightarrow e^{\prime}$ in CRWL if and only if $R\left(e, e^{\prime}\right) \rightarrow$ true in RL. In a similar way, strict equalities $a \bowtie b$ will be simulated through a binary relation $\bowtie$.

It just remains to translate the rules of deduction of the CRWL-calculus, which 
is straightforward. For example, the Bottom rule stating that every expression is reducible to $\perp$ is written

$$
R(x, \perp) \rightarrow \text { true if } \operatorname{pexpr}(x) \rightarrow \text { true },
$$

whereas the Join rule

$$
\frac{a \rightarrow t \quad b \rightarrow t \quad t \text { is a total term }}{a \bowtie b}
$$

results in

$$
x \bowtie y \rightarrow \text { true if } R(x, z) \rightarrow \operatorname{true} \wedge R(y, z) \rightarrow \operatorname{true} \wedge \operatorname{tterm}(z) \rightarrow \text { true } .
$$

Reflexivity, Transitivity, and Monotonicity are taken care of by, respectively:

$$
\begin{aligned}
& R(x, x) \rightarrow \text { true if } \operatorname{pexpr}(x) \rightarrow \text { true } \\
& R(x, y) \rightarrow \text { true if } R(x, z) \rightarrow \text { true } \wedge R(z, y) \rightarrow \text { true } \\
& R\left(h\left(x_{1}, \ldots, x_{n}\right), h\left(y_{1}, \ldots, y_{n}\right)\right) \rightarrow \text { true } \\
& \quad \text { if } R\left(x_{1}, y_{1}\right) \rightarrow \text { true } \wedge \ldots \wedge\left(x_{n}, y_{n}\right) \rightarrow \text { true } \quad\left(\forall h \in C_{\Sigma}^{n} \cup F_{\Sigma}^{n}, n \in \mathbb{N}\right)
\end{aligned}
$$

It is not necessary to include pexpr explicitly in all the rules because these conditions can be derived as logical consequences.

Finally, to every rule $l(\bar{v}) \rightarrow r(\bar{v}) \Leftarrow a_{1}(\bar{v}) \bowtie b_{1}(\bar{v}), \ldots, a_{m}(\bar{v}) \bowtie b_{m}(\bar{v})$ in the CRWL-theory, we associate the following rule in RL,

$$
\begin{aligned}
& R(l(\bar{x}), r(\bar{x})) \rightarrow \text { true } \\
& \quad \text { if } a_{1}(\bar{x}) \bowtie b_{1}(\bar{x}) \rightarrow \operatorname{true} \wedge \ldots \wedge a_{m}(\bar{x}) \bowtie b_{m}(\bar{x}) \rightarrow \operatorname{true} \wedge \\
& \quad \text { pterm }\left(x_{1}\right) \rightarrow \operatorname{true} \wedge \ldots \wedge \operatorname{term}\left(x_{n}\right) \rightarrow \text { true },
\end{aligned}
$$

where each CRWL variable $v_{i}$ (a constant in the RL-theory) has been replaced by the variable $x_{i}$. The set of all these last rules corresponds to the Reduction rule in the CRWL-calculus, and the condition that program rules in CRWL can only be instantiated with terms is taken care of by demanding pterm $(x) \rightarrow \operatorname{true}$ for all the variables appearing in it.

We will write $\alpha(T)=\left(\Sigma^{\prime}, \emptyset, \Gamma^{\prime}\right)$ for the RL-theory associated to a CRWL-theory $T$ in this fashion, with $\Sigma^{\prime}=\Sigma \cup \mathcal{V} \cup\{$ true, pterm, pexpr, tterm, $R, \bowtie, \perp\}$ and $\Gamma^{\prime}$ consisting of all the rules described above. The following proposition ensures that the translation is correct. Note that we use $=$ to denote both syntactic and semantic equality: the context will always make clear to which one we refer.

Proposition 2

For a CRWL-theory $T=(\Sigma, \Gamma)$ with $\alpha(T)=\left(\Sigma^{\prime}, \emptyset, \Gamma^{\prime}\right)$, if $l, r, a, b \in T_{\Sigma^{\prime}}(\mathcal{X})$ :

$l, r \in \operatorname{Expr}_{\perp}(\Sigma, \mathcal{V})$ and $T \vdash_{\mathrm{CRWL}} l \rightarrow r \Leftrightarrow \alpha(T) \vdash_{\mathrm{RL}} R(l, r) \rightarrow$ true; $a, b \in \operatorname{Expr}_{\perp}(\Sigma, \mathcal{V})$ and $T \vdash_{\mathrm{CRWL}} a \bowtie b \quad \Leftrightarrow \quad \alpha(T) \vdash_{\mathrm{RL}} a \bowtie b \rightarrow$ true.

The following two lemmas, which can be easily proved by structural induction on derivations, are needed for its proof. In particular, Lemma 1 is used in the most difficult part, which corresponds to Transitivity in the $(\Leftarrow)$-direction. 


\section{Lemma 1}

Let $T=(\Sigma, \Gamma)$ be a CRWL-theory, $\alpha(T)=\left(\Sigma^{\prime}, \emptyset, \Gamma^{\prime}\right)$, and $e, e^{\prime} \in T_{\Sigma^{\prime}}(\mathcal{X})$.

1. If $\alpha(T) \vdash_{\mathrm{RL}} e \rightarrow e^{\prime}$ and $e \in \operatorname{Expr}_{\perp}(\Sigma, \mathcal{V})$ or $e^{\prime} \in \operatorname{Exp}_{\perp}(\Sigma, \mathcal{V})$, then $e=e^{\prime}$.

2. If $\alpha(T) \vdash_{\mathrm{RL}} \operatorname{tterm}(e) \rightarrow e^{\prime}$, then $e^{\prime}$ is either true or tterm $\left(e^{\prime \prime}\right)$ for some $e^{\prime \prime}$ such that $\alpha(T) \vdash_{\mathrm{RL}} e \rightarrow e^{\prime \prime}$.

\section{Lemma 2}

If $T=(\Sigma, \Gamma)$ is a CRWL-theory, $\alpha(T)=\left(\Sigma^{\prime}, \emptyset, \Gamma^{\prime}\right)$, and $e \in T_{\Sigma^{\prime}}(\mathcal{X})$, then:

1. $e \in \operatorname{Term}(\Sigma, \mathcal{V}) \Leftrightarrow \alpha(T) \vdash_{\mathrm{RL}} \operatorname{tterm}(e) \rightarrow$ true,

2. $e \in \operatorname{Term}_{\perp}(\Sigma, \mathcal{V}) \Leftrightarrow \alpha(T) \vdash_{\mathrm{RL}} \operatorname{pterm}(e) \rightarrow$ true,

3. $e \in \operatorname{Expr}_{\perp}(\Sigma, \mathcal{V}) \Leftrightarrow \alpha(T) \vdash_{\mathrm{RL}} \operatorname{pexpr}(e) \rightarrow$ true.

\section{Proof of Proposition Q}

Both directions are proved by induction on the derivation, studying the last rule applied. Let us first consider the $(\Rightarrow)$ part.

- Bottom. We have $T \vdash_{\mathrm{CRWL}} l \rightarrow \perp$. Since $l \in \operatorname{Expr}_{\perp}(\Sigma, \mathcal{V})$, by Lemma 2 it is $\alpha(T) \vdash_{\mathrm{RL}} \operatorname{pexpr}(l) \rightarrow$ true so, by the translation of the Bottom rule, we have $\alpha(T) \vdash_{\mathrm{RL}} R(l, \perp) \rightarrow$ true.

- Reflexivity. By Lemma 2] $\alpha(T) \vdash_{\mathrm{RL}} \operatorname{pexpr}(l) \rightarrow$ true, so the result follows by applying the third rule associated to the relation $R$.

- Transitivity. We have that the last step in the derivation is

$$
\frac{a \rightarrow t \quad t \rightarrow r}{l \rightarrow r}
$$

By induction hypothesis, $\alpha(T) \vdash_{\mathrm{RL}} R(l, t) \rightarrow$ true and $\alpha(T) \vdash_{\mathrm{RL}} R(t, r) \rightarrow$ true, and by the fourth rule associated to $R$ we can derive $\alpha(T) \vdash_{\mathrm{RL}} R(l, r) \rightarrow$ true.

- Monotonicity. Similarly to the previous case.

- Join. From

$$
\frac{a \rightarrow t \quad b \rightarrow t}{a \bowtie b} \quad t \in \operatorname{Term}(\Sigma, \mathcal{V}),
$$

we get, by induction hypothesis, $\alpha(T) \vdash_{\mathrm{RL}} R(a, t) \rightarrow$ true and $\alpha(T) \vdash_{\mathrm{RL}} R(b, t) \rightarrow$ true, and by Lemma 2$] \alpha(T) \vdash_{\mathrm{RL}}$ tterm $(t) \rightarrow$ true, so we can apply the rule associated to $\bowtie$ to reach the result.

- Reduction. Assume that for some rule $l(\bar{v}) \rightarrow r(\bar{v}) \Leftarrow a_{1}(\bar{v}) \bowtie b_{1}(\bar{v}), \ldots, a_{m}(\bar{v}) \bowtie$ $b_{m}(\bar{v})$ in $\Gamma$ and partial terms $w_{1}, \ldots, w_{n}$, the last step in the derivation is

$$
\frac{a_{1}(\bar{w} / \bar{v}) \bowtie b_{1}(\bar{w} / \bar{v}) \ldots a_{m}(\bar{w} / \bar{v}) \bowtie b_{m}(\bar{w} / \bar{v})}{l(\bar{w} / \bar{v}) \rightarrow r(\bar{w} / \bar{v})} .
$$

Then, by induction hypothesis, $\alpha(T) \vdash a_{i}(\bar{w} / \bar{v}) \bowtie b_{i}(\bar{w} / \bar{v}) \rightarrow$ true for $i=1, \ldots, m$ and, by Lemma2 $\alpha(T) \vdash \operatorname{pexpr}\left(w_{i}\right) \rightarrow$ true for $i=1, \ldots, n$. Then the result follows by using the rule associated to $l(\bar{v}) \rightarrow r(\bar{v}) \Leftarrow a_{1}(\bar{v}) \bowtie b_{1}(\bar{v}), \ldots, a_{m}(\bar{v}) \bowtie b_{m}(\bar{v})$ in $\alpha(T)$.

Let us now consider the converse $(\Leftarrow)$. Note that even though the names of some of the rules are the same, the induction proceeds now over derivations in RL. 
- Reflexivity and Congruence are not possible.

- Transitivity. Assume that

$$
\frac{R(l, r) \rightarrow e \quad e \rightarrow \text { true }}{R(l, r) \rightarrow \text { true }} .
$$

(The case for $a \bowtie b$ is analogous.) By induction on the derivation of $\alpha(T) \vdash_{\mathrm{RL}}$ $R(l, r) \rightarrow e$ and using the fact that true only rewrites to itself, it follows easily that $e$ must be either true, or $R\left(l^{\prime}, r^{\prime}\right)$ with $\alpha(T) \vdash_{\mathrm{RL}} l \rightarrow l^{\prime}$ and $\alpha(T) \vdash_{\mathrm{RL}} r \rightarrow$ $r^{\prime}$. In the first case the result follows from the induction hypothesis applied to $R(l, r) \rightarrow e$. In the second, again by the induction hypothesis, $l^{\prime}, r^{\prime} \in \operatorname{Expr}_{\perp}(\Sigma, \mathcal{V})$ and $T \vdash_{\mathrm{CRWL}} l^{\prime} \rightarrow r^{\prime}$, and by Lemma 1 we have $l=l^{\prime}$ and $r=r^{\prime}$.

- Replacement. The result follows because the rules associated to the relation $R$ reflect faithfully the rules of deduction of CRWL. For example, consider the rule associated to Join. If

$$
\frac{R(a, c) \rightarrow \text { true } \quad R(b, c) \rightarrow \text { true } \quad \text { tterm }(c) \rightarrow \text { true }}{a \bowtie b \rightarrow \text { true }},
$$

then $c \in \operatorname{Term}(\Sigma, \mathcal{V})$ by Lemma 2 and $T \vdash_{\mathrm{CRWL}} a \rightarrow c$ and $T \vdash_{\mathrm{CRWL}} b \rightarrow c$ by the induction hypothesis, whence follows that $T \vdash_{\mathrm{CRWL}} a \bowtie b$.

\subsection{Simulating $R L$ in $C R W L$}

We now embark ourselves on finding the converse simulation of RL in CRWL. We are again interested in a computable and simple translation, and the idea for this is very similar to that of the previous section. Now, however, there are no terms and expressions to distinguish, and therefore predicates such as pterm are no longer necessary; as a consequence, we will be able to use the same set $\mathcal{X}$ of variables for both logics. The fact that only joinability statements are allowed to appear in the condition of a rewrite rule in CRWL forces us to represent, as in Section 2.6 the rewriting relation in RL through a binary relation $R$ in CRWL, so that $t \rightarrow t^{\prime}$ in $\mathrm{RL}$ if and only if $R\left(t, t^{\prime}\right) \rightarrow$ true in CRWL. Rewriting modulo a set of equations will be handled by transforming each equation $t=t^{\prime}$ into the rewrites $t \rightarrow t^{\prime}$ and $t^{\prime} \rightarrow t$.

More precisely, given a signature $(\Sigma, E)$ in RL we associate to it a CRWL-theory over the signature $\Sigma^{\prime}$ with $C_{\Sigma^{\prime}}=\Sigma \cup\{$ true $\}$ and $F_{\Sigma^{\prime}}=\{R\}$, with true and $R$ of arities 0 and 2 , respectively. The rules in the theory include

$$
\begin{aligned}
& R\left(x_{1}, x_{2}\right) \rightarrow \text { true } \Leftarrow x_{1} \bowtie x_{2}, \\
& R(x, y) \rightarrow \text { true } \Leftarrow R(x, z) \bowtie \text { true }, R(z, y) \bowtie \text { true },
\end{aligned}
$$

and, for each $f \in \Sigma$ of arity $n \in \mathbb{N}$,

$$
\begin{aligned}
& R\left(f\left(x_{1}, \ldots, x_{n}\right), f\left(y_{1}, \ldots, y_{n}\right)\right) \rightarrow \text { true } \\
& \qquad R\left(x_{1}, y_{1}\right) \bowtie \text { true }, \ldots, R\left(x_{n}, y_{n}\right) \bowtie \text { true },
\end{aligned}
$$

mimicking the Reflexivity, Transitivity, and Congruence rules in the RLcalculus, together with

$$
\begin{aligned}
& R\left(t, t^{\prime}\right) \rightarrow \text { true }, \\
& R\left(t^{\prime}, t\right) \rightarrow \text { true },
\end{aligned}
$$


for every $t=t^{\prime} \in E$. The goal of the condition in the rule corresponding to Reflexivity is to avoid instantiating it with terms containing $\perp$, which have no meaning in RL.

A conditional rewrite rule

$$
[l] \rightarrow[r] \text { if }\left[a_{1}\right] \rightarrow\left[b_{1}\right] \wedge \ldots \wedge\left[a_{m}\right] \rightarrow\left[b_{m}\right]
$$

over $(\Sigma, E)$ in $\mathrm{RL}$ is then translated to

$$
R(l, r) \rightarrow \text { true } \Leftarrow R\left(a_{1}, b_{1}\right) \bowtie \text { true }, \ldots, R\left(a_{m}, b_{m}\right) \bowtie \text { true },
$$

where $l, r, a_{i}, b_{i}$ are arbitrary members of $[l],[r],\left[a_{i}\right]$, and $\left[b_{i}\right]$, respectively. Then, given an RL-theory $T=(\Sigma, E, \Gamma)$ we associate to it the CRWL-theory $\beta(T)$ obtained by adding to the CRWL-theory corresponding to $(\Sigma, E)$ the translation of the rules in $\Gamma$.

Actually, the previous definitions must be slightly modified due to some technical details. Recall from Section [2.3] that in a conditional rewrite rule $l \rightarrow r \Leftarrow C$ in CRWL $l$ must be linear, and it is obvious that with the above definitions this property is not ensured for the translation of equations and rewrite rules; therefore, those rules must be "linearised" (Arenas-Sánchez and Rodríguez-Artalejo 2001). The linearised version of a conditional rewrite rule $l \rightarrow r \Leftarrow C$ is given by $l^{\prime} \rightarrow r \Leftarrow C, C_{l}$, where $l^{\prime}$ and $C_{l}$ are calculated as follows: for every variable $x$ appearing $k>1$ times in $l$, its $j$-th occurrence, $2 \leq j \leq k$, is replaced with a new variable $y_{j}$ and $x \bowtie y_{j}$ is added to $C_{l}$. Moreover (and this is simply a feature of our translation), even when a variable $x$ appears only once, $x \bowtie x$ will be added to the conditional part so that $x$ cannot be instantiated with a partial term. The treatment of linearised rules in the rest of the section, though rigorous, will not be too formal.

The following proposition shows that our translation correctly reflects provability in the equational part of a rewrite theory.

Proposition 3

If $\left(\Sigma^{\prime}, E^{\prime}\right)$ is the CRWL theory corresponding to a signature $(\Sigma, E)$ in RL and if $t, t^{\prime} \in T_{\Sigma}(\mathcal{X})$ are such that $E \vdash t=t^{\prime}$, then

$$
E^{\prime} \vdash_{\mathrm{CRWL}} R\left(t, t^{\prime}\right) \rightarrow \text { true } \quad \text { and } \quad E^{\prime} \vdash_{\mathrm{CRWL}} R\left(t^{\prime}, t\right) \rightarrow \text { true } .
$$

\section{Proof}

By induction on the derivation of $E \vdash t=t^{\prime}$. The rules of a deduction system for equational logic include those in Figure 1 (replacing the arrow with an equality symbol) together with a Symmetry rule. Let us just consider the case of the Replacement rule. Since our equational logic is unconditional, we have

$$
\overline{\theta\left(t_{1}\right)=\theta\left(t_{2}\right)} \quad\left(t_{1}=t_{2}\right) \in E
$$

for some assignment $\theta: \mathcal{X} \rightarrow T_{\Sigma}(\mathcal{X})$. Associated to $t_{1}=t_{2}$ we have the linearised versions of the two rules $R\left(t_{1}, t_{2}\right) \rightarrow$ true and $R\left(t_{2}, t_{1}\right) \rightarrow$ true in $E^{\prime}$ and, since $T_{\Sigma}(\mathcal{X}) \subseteq \operatorname{Term}_{\perp}\left(\Sigma^{\prime}, \mathcal{X}\right)$, we can instantiate them with $\theta$ (mapping those $x$ which arose in the linearization process to the same term as the original variable) to obtain the result. 
With this in hand we are ready to prove the first half of the main proposition, which guarantees the correctness of the translation.

Proposition 4

Given any RL-theory $T=(\Sigma, E, \Gamma)$, and $l, r \in T_{\Sigma}(\mathcal{X})$ :

$$
\begin{aligned}
T \vdash_{\mathrm{RL}}[l] \rightarrow[r] & \Rightarrow\left(\exists l^{\prime} \in[l], \exists r^{\prime} \in[r]\right) \beta(T) \vdash_{\mathrm{CRWL}} R\left(l^{\prime}, r^{\prime}\right) \rightarrow \text { true } \\
& \Leftrightarrow \quad\left(\forall l^{\prime} \in[l], \forall r^{\prime} \in[r]\right) \beta(T) \vdash_{\mathrm{CRWL}} R\left(l^{\prime}, r^{\prime}\right) \rightarrow \text { true }
\end{aligned}
$$

Proof

Let us first prove the equivalence. There is nothing to prove in the right-to-left direction; in the opposite one, the result is a consequence of Proposition 3 and the rule $R(x, y) \rightarrow$ true $\Leftarrow R(x, z) \bowtie$ true,$R(z, y) \bowtie$ true that, by construction, is in $\beta(T)$. Now we prove the first implication by induction on the derivation, according to the last rule used:

- Reflexivity. $T \vdash_{\mathrm{RL}}[l] \rightarrow[l]$, and the result follows by instantiating $R\left(x_{1}, x_{2}\right) \rightarrow$ true $\Leftarrow x_{1} \bowtie x_{2}$ with $l$ for both variables.

- Congruence. From

$$
\frac{\left[l_{1}\right] \rightarrow\left[r_{1}\right] \quad \ldots \quad\left[l_{n}\right] \rightarrow\left[r_{n}\right]}{\left[f\left(l_{1}, \ldots, l_{n}\right)\right] \rightarrow\left[f\left(r_{1}, \ldots, r_{n}\right)\right]}
$$

and the induction hypothesis, $\beta(T) \vdash_{\mathrm{CRWL}} R\left(l_{i}^{\prime}, r_{i}^{\prime}\right) \rightarrow$ true for some $l_{i}^{\prime} \in\left[l_{i}\right]$, $r_{i}^{\prime} \in\left[r_{i}\right], 1 \leq i \leq n$. Then, using the rule $R\left(f\left(x_{1}, \ldots, x_{n}\right), f\left(y_{1}, \ldots, y_{n}\right)\right) \rightarrow$ true $\Leftarrow$ $R\left(x_{1}, y_{1}\right) \bowtie$ true $, \ldots, R\left(x_{n}, y_{n}\right) \bowtie$ true, we get

$$
\beta(T) \vdash_{\mathrm{CRWL}} R\left(f\left(l_{1}^{\prime}, \ldots, l_{n}^{\prime}\right), f\left(r_{1}^{\prime}, \ldots, r_{n}^{\prime}\right)\right) \rightarrow \text { true },
$$

verifying $f\left(l_{1}^{\prime}, \ldots, l_{n}^{\prime}\right) \in\left[f\left(l_{1}, \ldots, l_{n}\right)\right]$ and $f\left(r_{1}^{\prime}, \ldots, r_{n}^{\prime}\right) \in\left[f\left(r_{1}, \ldots, r_{n}\right)\right]$.

\section{- Transitivity. From}

$$
\frac{[l] \rightarrow[t] \quad[t] \rightarrow[r]}{[l] \rightarrow[r]}
$$

and the induction hypothesis, $\beta(T) \vdash_{\mathrm{CRWL}} R\left(l^{\prime}, t^{\prime}\right) \rightarrow$ true and $\beta(T) \vdash_{\mathrm{CRWL}}$ $R\left(t^{\prime \prime}, r^{\prime}\right) \rightarrow$ true, with $l^{\prime} \in[l], t^{\prime}, t^{\prime \prime} \in[t]$, and $r^{\prime} \in[r]$. Then, due to the equivalence proved above, $\beta(T) \vdash_{\mathrm{CRWL}} R(l, t) \rightarrow$ true and $\beta(T) \vdash_{\mathrm{CRWL}} R(t, r) \rightarrow$ true and we get the result using the translation of the Transitivity rule.

- Replacement. We have, for some $[l(\bar{x})] \rightarrow[r(\bar{x})]$ if $\left[a_{1}(\bar{x})\right] \rightarrow\left[b_{1}(\bar{x})\right] \wedge \ldots \wedge$ $\left[a_{m}(\bar{x})\right] \rightarrow\left[b_{m}(\bar{x})\right]$ in $\Gamma$,

$$
\begin{aligned}
& {\left[w_{1}\right] \rightarrow\left[w_{1}^{\prime}\right] \quad \ldots \quad\left[w_{n}\right] \rightarrow\left[w_{n}^{\prime}\right]} \\
& \frac{\left[a_{1}(\bar{w} / \bar{x})\right] \rightarrow\left[b_{1}(\bar{w} / \bar{x})\right] \quad \ldots \quad\left[a_{m}(\bar{w} / \bar{x})\right] \rightarrow\left[b_{m}(\bar{w} / \bar{x})\right]}{T \vdash_{\mathrm{RL}}[l(\bar{w} / \bar{x})] \rightarrow\left[r\left(\overline{w^{\prime}} / \bar{x}\right)\right]} .
\end{aligned}
$$

By induction hypothesis, there exist $a_{i}^{\prime} \in\left[a_{i}(\bar{w} / \bar{x})\right], b_{i}^{\prime} \in\left[b_{i}(\bar{w} / \bar{x})\right]$ such that $\beta(T) \vdash_{\mathrm{CRWL}} R\left(a_{i}^{\prime}, b_{i}^{\prime}\right) \rightarrow$ true for $i=1, \ldots, m$. Again by the equivalence shown above, $\beta(T) \vdash_{\mathrm{CRWL}} R\left(a_{i}(\bar{w} / \bar{x}), b_{i}(\bar{w} / \bar{x})\right) \rightarrow$ true, for $i=1, \ldots, m$. We can then use the linearised version of $R(l, r) \rightarrow$ true $\Leftarrow R\left(a_{1}, b_{1}\right) \bowtie$ true $, \ldots, R\left(a_{m}, b_{m}\right) \bowtie$ true, substituting all variables which arose from the same one during the linearisation process with the same $w_{i}$ (so that the conditions $x \bowtie x, x \bowtie y_{j}$ are 
trivially verified), to get $\beta(T) \vdash_{\mathrm{CRWL}} R(l(\bar{w} / \bar{x}), r(\bar{w} / \bar{x})) \rightarrow$ true. In a similar way, $\beta(T) \vdash_{\mathrm{CRWL}} R\left(w_{i}, w_{i}^{\prime}\right) \rightarrow$ true, $i=1, \ldots, n$, is also obtained, and repeated aplication of the translation of the Transitivity and Congruence rules would show, first, that $\beta(T) \vdash_{\mathrm{CRWL}} R\left(r(\bar{w} / \bar{x}), r\left(\overline{w^{\prime}} / \bar{x}\right)\right) \rightarrow$ true, and then $\beta(T) \vdash_{\mathrm{CRWL}}$ $R\left(l(\bar{w} / \bar{x}), r\left(\overline{w^{\prime}} / \bar{x}\right)\right) \rightarrow$ true, as desired.

Our next goal will be to prove the converse of the last proposition. However, more care is needed here since, for example, an equation of the form $x * 0=0$ will allow us to derive $R($ true $* 0,0) \rightarrow$ true. Even more bizarre derivations are possible by repeated application of transitivity, e.g. $R(R($ true, true $)$, true $) \rightarrow$ true. To prove that these rewrites, however, do not allow us to derive anything in CRWL that was not already derivable in the original RL-theory, we concentrate first on some preliminary results. The first one is proved by an easy induction for each fact in the statement.

\section{Lemma 3}

Let $T=(\Sigma, E, \Gamma)$ be an RL-theory, $\beta(T)=\left(\Sigma^{\prime}, \Gamma^{\prime}\right)$, and $e, e_{1}, \ldots, e_{n} \in \operatorname{Expr}_{\perp}\left(\Sigma^{\prime}, \mathcal{X}\right)$ expressions in CRWL.

1. If $\beta(T) \vdash_{\mathrm{CRWL}} \perp \rightarrow e$, then $e=\perp$.

2. For all $x \in \mathcal{X}$, if $\beta(T) \vdash_{\text {CRWL }} x \rightarrow e$, then either $e=\perp$ or $e=x$.

3. If $\beta(T) \vdash_{\text {CRWL }} R\left(e_{1}, e_{2}\right) \rightarrow e$, then either $e=\perp$, or $e=$ true, or $e=R\left(e_{1}^{\prime}, e_{2}^{\prime}\right)$ with $\beta(T) \vdash_{\mathrm{CRWL}} e_{i} \rightarrow e_{i}^{\prime}, i=1,2$.

4. For every $f \in C_{\Sigma^{\prime}}^{n}$, if $\beta(T) \vdash_{\text {CRWL }} f\left(e_{1}, \ldots, e_{n}\right) \rightarrow e$ then either $e=\perp$, or $e=f\left(e_{1}^{\prime}, \ldots, e_{n}^{\prime}\right)$ with $\beta(T) \vdash_{\mathrm{CRWL}} e_{i} \rightarrow e_{i}^{\prime}$ for some $e_{i}^{\prime} \in \operatorname{Expr}_{\perp}\left(\Sigma^{\prime}, \mathcal{X}\right)$, $i=1, \ldots, n$.

In what follows this lemma will be used mostly without explicit reference to it: for example, when deducing $\beta(T) \vdash_{\mathrm{CRWL}} R\left(t, t^{\prime}\right) \rightarrow$ true from $\beta(T) \vdash_{\mathrm{CRWL}} R\left(t, t^{\prime}\right) \bowtie$ true.

\section{Lemma 4}

Let $T$ be an RL-theory and $\beta(T)=\left(\Sigma^{\prime}, \Gamma^{\prime}\right)$.

1. For all $e, e^{\prime} \in \operatorname{Expr}_{\perp}\left(\Sigma^{\prime}, \mathcal{X}\right)$, if $\beta(T) \vdash_{\mathrm{CRWL}} e \rightarrow e^{\prime}$ and $e^{\prime}$ is total, then $e$ is total.

2. For all $t \in \operatorname{Term}_{\perp}\left(\Sigma^{\prime}, \mathcal{X}\right), e^{\prime} \in \operatorname{Expr}_{\perp}(\Sigma, \mathcal{X})$, if $\beta(T) \vdash_{\mathrm{CRWL}} t \rightarrow e^{\prime}$ and $e^{\prime}$ is total, then $t=e^{\prime}$.

3. For all $t, t^{\prime} \in \operatorname{Term}_{\perp}\left(\Sigma^{\prime}, \mathcal{X}\right)$, if $\beta(T) \vdash_{\mathrm{CRWL}} t \bowtie t^{\prime}$, then $t$ is total and $t^{\prime}=t$.

Proof

1. By induction on the last rule of the derivation. Rules Bottom and Join are not possible, and Reflexivity is immediate. For Transitivity, if the last step of the derivation is

$$
\frac{e \rightarrow e^{\prime \prime} \quad e^{\prime \prime} \rightarrow e^{\prime}}{e \rightarrow e^{\prime}}
$$

then, by induction hypothesis, $e^{\prime \prime}$ is total, and again by induction hypothesis $e$ is total. The situation is similar for Monotonicity. For Reduction we have to distinguish all these cases: 
- If $R\left(x_{1}, x_{2}\right) \rightarrow$ true $\Leftarrow x_{1} \bowtie x_{2}$ has been used, then $e=R\left(e_{1}, e_{2}\right)$ and $\beta(T) \vdash_{\mathrm{CRWL}} e_{1} \rightarrow t, \beta(T) \vdash_{\mathrm{CRWL}} e_{2} \rightarrow t$ for some $t$, total, have been previously obtained in the derivation. By induction hypothesis, both $e_{1}$ and $e_{2}$ are total and so is $e$.

- If a rule of the form $R\left(f\left(x_{1}, \ldots, x_{n}\right), f\left(y_{1}, \ldots, y_{n}\right)\right) \rightarrow \operatorname{true} \Leftarrow R\left(x_{1}, y_{1}\right) \bowtie$ true $, \ldots, R\left(x_{n}, y_{n}\right) \bowtie$ true or $R(x, y) \rightarrow$ true $\Leftarrow R(x, z) \bowtie \operatorname{true}, R(z, y) \bowtie$ true has been used, the result follows by induction hypothesis.

- If the last rule applied has been one of those corresponding to equations or rewrite rules then $e=R(l, r)$ and a condition of the form $x \bowtie x$ or $x \bowtie y_{j}$ for every variable appearing in it must have been satisfied. If $x$ has been instantiated with $t$, then those conditions imply that $\beta(T) \vdash_{\mathrm{CRWL}} t \rightarrow t^{\prime}$ for some total $t^{\prime}$, so by induction hypothesis $t$ is total, and so will be the expression $e$.

2. By (1), $t \in \operatorname{Term}\left(\Sigma^{\prime}, \mathcal{X}\right)$. By structural induction on $t$ :

- $t=x$, then $e^{\prime}=\perp$ (absurd) or $e^{\prime}=x$ and the result holds;

- $t=f\left(t_{1}, \ldots, t_{n}\right)$, then either $e^{\prime}=\perp$ (absurd) or $e^{\prime}=f\left(e_{1}^{\prime}, \ldots, e_{n}^{\prime}\right)$ with $\beta(T) \vdash_{\mathrm{CRWL}} t_{i} \rightarrow e_{i}^{\prime}$. In this last case, by induction hypothesis, $t_{i}=e_{i}^{\prime}$ for $i=1, \ldots, n$ and so $t=e^{\prime}$.

3. There exists $t^{\prime \prime} \in \operatorname{Term}\left(\Sigma^{\prime}, \mathcal{X}\right)$ with $\beta(T) \vdash_{\mathrm{CRWL}} t \rightarrow t^{\prime \prime}$ and $\beta(T) \vdash_{\mathrm{CRWL}} t^{\prime} \rightarrow$ $t^{\prime \prime}$, and by $(2), t=t^{\prime \prime}=t^{\prime}$.

We are now ready to prove our goal and we will do so in two steps. The next proposition shows that if $R(l, r) \rightarrow$ true can be proved in $\beta(T)$ then $[l] \rightarrow[r]$ can also be proved in $T$, but extending the signature with the constant true. After that, we show that we can forget about this extra constant.

Proposition 5

Let $T=(\Sigma, E, \Gamma)$ be an RL-theory, $\beta(T)=\left(\Sigma^{\prime}, \Gamma^{\prime}\right)$, and let $l, r \in \operatorname{Term}_{\perp}\left(\Sigma^{\prime}, \mathcal{X}\right)$. If $\beta(T) \vdash_{\text {CRWL }} R(l, r) \rightarrow$ true then $l, r \in \operatorname{Term}\left(\Sigma^{\prime}, \mathcal{X}\right)$ (recall that $\operatorname{Term}\left(\Sigma^{\prime}, \mathcal{X}\right)=$ $\left.T_{\Sigma \cup\{\text { true }\}}(\mathcal{X})\right)$ and $(\Sigma \cup\{$ true $\}, E, \Gamma) \vdash_{\mathrm{RL}}[l] \rightarrow[r]$.

Proof

By Lemma 41$] l, r \in \operatorname{Term}\left(\Sigma^{\prime}, \mathcal{X}\right)$. For the second part, we proceed by induction on the proof of $\beta(T) \vdash_{\text {CRWL }} R(l, r) \rightarrow$ true. The last rule applied must have been Transitivity or Reduction.

- For Transitivity we have

$$
\frac{R(l, r) \rightarrow e \quad e \rightarrow \text { true }}{R(l, r) \rightarrow \text { true }}
$$

If $e=$ true the result follows by induction hypothesis. Otherwise it must be $e=$ $R\left(l^{\prime}, r^{\prime}\right)$, total by Lemma 41] with $\beta(T) \vdash_{\mathrm{CRWL}} l \rightarrow l^{\prime}$ and $\beta(T) \vdash_{\mathrm{CRWL}} r \rightarrow r^{\prime}$. Hence, by Lemma $42 l=l^{\prime}, r=r^{\prime}$, and the result holds by induction hypothesis.

- For Reduction there are five different cases, corresponding to each of the conditional rewrite rules simulating the RL-calculus in $\beta(T)$. Recall that rules in CRWL are instantiated only with members of $\operatorname{Term}_{\perp}\left(\Sigma^{\prime}, \mathcal{X}\right)$. 
1. If the last step of the derivation is

$$
\frac{l \bowtie r}{R(l, r) \rightarrow \text { true }}
$$

then by Lemma 43] it is $l=r$ and therefore $(\Sigma \cup\{t r u e\}, E, \Gamma) \vdash_{\mathrm{RL}}[l] \rightarrow[r]$.

2. If we have

$$
\frac{R(l, t) \bowtie \text { true } \quad R(t, r) \bowtie \text { true }}{R(l, r) \rightarrow \text { true }},
$$

then, by induction hypothesis, $(\Sigma \cup\{t r u e\}, E, \Gamma) \vdash_{\mathrm{RL}}[l] \rightarrow[t]$ and $(\Sigma \cup$ $\{$ true $\}, E, \Gamma) \vdash_{\mathrm{RL}}[t] \rightarrow[r]$, so $(\Sigma \cup\{$ true $\}, E, \Gamma) \vdash_{\mathrm{RL}}[l] \rightarrow[r]$ by Transitivity of RL.

3. For the translation of the Congruence rule the result also follows immediately by the induction hypothesis.

4. Assume that the result is obtained by using one of the (linearised) rules associated to an equation $t=t^{\prime} \in E$. The conditions of the form $x \bowtie x$ and $x \bowtie y_{j}$ in the rule together with Lemma 43 imply that all the variables which arose from the same one must have been instantiated with the same element of $\operatorname{Term}\left(\Sigma^{\prime}, \mathcal{X}\right)$. This way $E \vdash l=r$, so $[l]=[r]$ and $(\Sigma \cup\{$ true $\}, E, \Gamma) \vdash_{\mathrm{RL}}$ $[l] \rightarrow[r]$ by Reflexivity of RL.

5. If the last rule applied is one of those associated to an element of $\Gamma$ then, as in the previous case, all variables have been instantiated properly and the result follows by the induction hypothesis and using Replacement.

These results, combined with the completeness of RL, are enough to prove the converse of Proposition 4 again, since the semantics of RL is not explained until Section 3.2 we postpone the proof to the appendix.

Proposition 6

Given any RL-theory $T=(\Sigma, E, \Gamma)$, and $l, r \in T_{\Sigma}(\mathcal{X})$ :

$$
\begin{aligned}
T \vdash_{\mathrm{RL}}[l] \rightarrow[r] & \Leftrightarrow \quad\left(\exists l^{\prime} \in[l], \exists r^{\prime} \in[r]\right) \beta(T) \vdash_{\mathrm{CRWL}} R\left(l^{\prime}, r^{\prime}\right) \rightarrow \text { true } \\
& \Leftrightarrow \quad\left(\forall l^{\prime} \in[l], \forall r^{\prime} \in[r]\right) \beta(T) \vdash_{\mathrm{CRWL}} R\left(l^{\prime}, r^{\prime}\right) \rightarrow \text { true }
\end{aligned}
$$

\section{Relations at the Semantic Level}

In this section we leave behind our study of the entailment relations and turn our attention to models and satisfaction. Our interest lies in associating suitable institutions to both CRWL and RL and, thereafter, to relate them via maps of institutions with "good" properties.

\subsection{Institutions}

The notion of model is based on Goguen and Burstall's work on institutions (Goguen and Burstall 1992). An institution is a 4 -tuple $\mathcal{I}=(\operatorname{Sign}$, sen, Mod, $\models)$ such that:

- Sign is a category whose objects are called signatures. 
- sen $:$ Sign $\rightarrow$ Set is a functor associating to each signature $\Sigma$ a set of $\Sigma$ sentences.

- Mod : Sign ${ }^{\text {op }} \rightarrow$ Cat is a functor that gives for each signature $\Sigma$ a category whose objects are called $\Sigma$-models.

- $\models$ is a function associating to each $\Sigma \in|\mathbf{S i g n}|$ a binary relation $\models_{\Sigma} \subseteq$ $|\operatorname{Mod}(\Sigma)| \times \operatorname{sen}(\Sigma)$ called $\Sigma$-satisfaction, in such a way that the following property holds for any $H: \Sigma \rightarrow \Sigma^{\prime}, M^{\prime} \in\left|\operatorname{Mod}\left(\Sigma^{\prime}\right)\right|$, and all $\varphi \in \operatorname{sen}(\Sigma)$ :

$$
M^{\prime} \models_{\Sigma^{\prime}} \operatorname{sen}(H)(\varphi) \Leftrightarrow \operatorname{Mod}(H)\left(M^{\prime}\right) \models_{\Sigma} \varphi
$$

Given a set $\Gamma$ of $\Sigma$-sentences, the category $\operatorname{Mod}(\Sigma, \Gamma)$ is defined as the full subcategory of $\operatorname{Mod}(\Sigma)$ determined by those models $M \in|\operatorname{Mod}(\Sigma)|$ that satisfy all the sentences in $\Gamma$. A relation between sets of sentences and sentences, also denoted as $\models$, can be defined by

$$
\Gamma \models_{\Sigma} \varphi \Leftrightarrow M \models_{\Sigma} \varphi \text { for each } M \in|\operatorname{Mod}(\Sigma, \Gamma)| \text {. }
$$

We can then associate an entailment system to each institution $\mathcal{I}=$ (Sign, sen, $\operatorname{Mod}, \models)$ in a natural way by means of the triple $\mathcal{I}^{+}=(\operatorname{Sign}$, sen, $\models)$, where $\models$ now denotes the previously defined relation between sets of sentences and sentences; $\mathcal{I}^{+}$is easily seen to satisfy the conditions to be an entailment system.

Given an institution $\mathcal{I}$, its category $\mathbf{T h}$ of theories is defined as the category of theories associated to the entailment system $\mathcal{I}^{+}$. If $H:(\Sigma, \Gamma) \rightarrow\left(\Sigma^{\prime}, \Gamma^{\prime}\right)$ is a theory morphism and $M^{\prime} \in \operatorname{Mod}\left(\Sigma^{\prime}, \Gamma^{\prime}\right)$, it is not difficult to check that $\operatorname{Mod}(H)\left(M^{\prime}\right) \in$ $\operatorname{Mod}(\Sigma, \Gamma)$. The model functor Mod can then be extended to a functor Mod : $\mathbf{T h}^{\text {op }} \rightarrow$ Cat.

There are many different notions of morphisms between institutions in the literature; a good survey can be found in Goguen and Roşu (2002). Although it will not play a crucial role in what follows, to give the reader a flavour of the idea we present here the definition introduced in Meseguer (1989). Given institutions $\mathcal{I}=($ Sign, sen, Mod, $\models)$ and $\mathcal{I}^{\prime}=\left(\mathbf{S i g n}^{\prime}, \operatorname{sen}^{\prime}, \mathbf{M o d}^{\prime}, \models^{\prime}\right)$, a map of institutions $(\Phi, \alpha, \beta): \mathcal{I} \rightarrow \mathcal{I}^{\prime}$ consists of a natural transformation $\alpha:$ sen $\Rightarrow \operatorname{sen}^{\prime} \circ \Phi$, an $\alpha$ sensible functor ${ }^{1} \Phi: \mathbf{T h}_{0} \rightarrow \mathbf{T h}_{0}^{\prime}$, and a natural transformation $\beta: \mathbf{M o d}^{\prime} \circ \Phi^{\mathrm{op}} \Rightarrow$ Mod such that for each $\Sigma \in|\operatorname{Sign}|, \varphi \in \operatorname{sen}(\Sigma)$, and $M^{\prime} \in\left|\operatorname{Mod}^{\prime}(\Phi(\Sigma, \emptyset))\right|$ the following property is satisfied:

$$
M^{\prime} \models_{\Sigma^{\prime}}^{\prime} \alpha_{\Sigma}(\varphi) \Leftrightarrow \beta_{(\Sigma, \emptyset)}\left(M^{\prime}\right) \models_{\Sigma} \varphi
$$

\subsection{The models of $R L$}

Before proceeding to $\mathcal{R}$-systems, the models of $\mathrm{RL}$, we need the categorical notion of subequalizer (Lambek 1970), a notion generalizing that of equalizer of two functors. ${ }^{2}$

\footnotetext{
${ }^{1}$ Essentially, this means that $\Phi$ is determined by its restriction to empty theories and $\alpha$.

${ }^{2}$ In Miyoshi (1996), subequalizers are shown to coincide with inserters, a special kind of weighted limit, in the 2-category Cat. This allows the author to generalize the models of RL, building them over arbitrary 2-categories and even enriched categories.
} 
Given a family of pairs of functors $\left\{F_{i}, G_{i}: \mathcal{A} \rightarrow \mathcal{B}_{i} \mid i \in I\right\}$, the (simultaneous) subequalizer of this family is a category $\operatorname{Subeq}\left(\left(F_{i}, G_{i}\right)_{i \in I}\right)$ together with a functor

$$
J: \operatorname{Subeq}\left(\left(F_{i}, G_{i}\right)_{i \in I}\right) \rightarrow \mathcal{A}
$$

and a family of natural transformations $\left\{\alpha_{i}: F_{i} \circ J \Rightarrow G_{i} \circ J \mid i \in I\right\}$ satisfying the following universal property: given a functor $H: \mathcal{C} \rightarrow \mathcal{A}$ and a family of natural transformations $\left\{\beta_{i}: F_{i} \circ H \Rightarrow G_{i} \circ H \mid i \in I\right\}$, there exists a unique functor $\left(H,\left\{\beta_{i}\right\}_{i \in I}\right): \mathcal{C} \rightarrow \operatorname{Subeq}\left(\left(F_{i}, G_{i}\right)_{i \in I}\right)$ such that

$$
J \circ\left(H,\left\{\beta_{i}\right\}_{i \in I}\right)=H \quad \text { and } \quad \alpha_{i} \circ\left(H,\left\{\beta_{i}\right\}_{i \in I}\right)=\beta_{i} \quad(i \in I) .
$$

The construction of $\operatorname{Subeq}\left(\left(F_{i}, G_{i}\right)_{i \in I}\right)$ is quite simple. Its objects are pairs (A, $\left.\left\{b_{i}\right\}_{i \in I}\right)$ with $A$ an object in $\mathcal{A}$ and $b_{i}: F_{i}(A) \rightarrow G_{i}(A)$ a morphism in $\mathcal{B}_{i}$. Morphisms $a:\left(A,\left\{b_{i}\right\}_{i \in I}\right) \rightarrow\left(A^{\prime},\left\{b_{i}^{\prime}\right\}_{i \in I}\right)$ are morphisms $a: A \rightarrow A^{\prime}$ in $\mathcal{A}$ such that for each $i \in I, G_{i}(a) \circ b_{i}=b_{i}^{\prime} \circ F_{i}(a)$. The functor $J$ is just projection into the first component. The natural transformations $\alpha_{j}$ are defined by

$$
\alpha_{j}\left(A,\left\{b_{i}\right\}_{i \in I}\right)=b_{j} \quad(j \in I) .
$$

Then, given an RL-theory $\mathcal{R}=(\Sigma, E, L, \Gamma)$, an $\mathcal{R}$-system $\mathcal{S}$ is a category $\mathcal{S}$ together with:

- A $(\Sigma, E)$-algebra structure given by a family of functors

$$
\left\{f_{\mathcal{S}}: \mathcal{S}^{n} \rightarrow \mathcal{S} \mid f \in \Sigma \text { of arity } n\right\}
$$

satisfying the equations $E$, i.e., for any $t\left(x_{1}, \ldots, x_{n}\right)=t^{\prime}\left(x_{1}, \ldots, x_{n}\right)$ in $E$ we have an identity of functors $t_{\mathcal{S}}=t_{\mathcal{S}}^{\prime}$, where the functor $t_{\mathcal{S}}$ is defined inductively from the functors $f_{\mathcal{S}}$ in the obvious way.

- For each rewrite rule

$$
r:[t(\bar{x})] \rightarrow\left[t^{\prime}(\bar{x})\right] \text { if }\left[a_{1}(\bar{x})\right] \rightarrow\left[b_{1}(\bar{x})\right] \wedge \ldots \wedge\left[a_{m}(\bar{x})\right] \rightarrow\left[b_{m}(\bar{x})\right]
$$

in $\Gamma$, a natural transformation

$$
r_{\mathcal{S}}: t_{\mathcal{S}} \circ J_{\mathcal{S}} \Rightarrow t_{\mathcal{S}}^{\prime} \circ J_{\mathcal{S}}
$$

where $J_{\mathcal{S}}: \operatorname{Subeq}\left(\left(a_{j \mathcal{S}}, b_{j \mathcal{S}}\right)_{1 \leq j \leq m}\right) \rightarrow \mathcal{S}^{n}$ is the subequalizer functor.

An $\mathcal{R}$-homomorphism $F: \mathcal{S} \rightarrow \mathcal{S}^{\prime}$ between two $\mathcal{R}$-systems is then a functor $F$ : $\mathcal{S} \rightarrow \mathcal{S}^{\prime}$ such that:

- It is a $\Sigma$-algebra homomorphism, i.e., $F \circ f_{\mathcal{S}}=f_{\mathcal{S}^{\prime}} \circ F^{n}$, for each $f$ in $\Sigma$ of arity $n$.

- " $F$ preserves $\Gamma$," i.e., for each rewrite rule $r:[t(\bar{x})] \rightarrow\left[t^{\prime}(\bar{x})\right]$ if $C$ in $\Gamma$ we have the identity of natural transformations

$$
F \circ r_{\mathcal{S}}=r_{\mathcal{S}^{\prime}} \circ F^{\bullet}
$$

where $F^{\bullet}: \operatorname{Subeq}\left(C_{\mathcal{S}}\right) \rightarrow \operatorname{Subeq}\left(C_{\mathcal{S}^{\prime}}\right)$ is the unique functor induced by the universal property of $\operatorname{Subeq}\left(C_{\mathcal{S}^{\prime}}\right)$ by the composition functor

$$
\operatorname{Subeq}\left(C_{\mathcal{S}}\right) \stackrel{J_{\mathcal{S}}}{\longrightarrow} \mathcal{S}^{n} \stackrel{F^{n}}{\longrightarrow} \mathcal{S}^{\prime n}
$$


and the natural transformations $F \circ \alpha_{j}, 1 \leq j \leq m$, where the condition $C$ has $m$ rewrites $\left[a_{j}\right] \rightarrow\left[b_{j}\right]$, and $\alpha_{j}$ is the $j$ th natural transformation associated to the subequalizer $\operatorname{Subeq}\left(C_{\mathcal{S}}\right)$. Despite the somewhat complicated definition of $F^{\bullet}$, its behavior on objects is quite simple; it is given by the equation

$$
F^{\bullet}\left(\bar{C}^{n}, \bar{c}^{m}\right)=\left(F^{n}\left(\bar{C}^{n}\right), F^{m}\left(\bar{c}^{m}\right)\right) .
$$

This defines a category $\mathcal{R}$-Sys in the obvious way.

A sequent $\left[t\left(x_{1}, \ldots, x_{n}\right)\right] \rightarrow\left[t^{\prime}\left(x_{1}, \ldots, x_{n}\right)\right]$ is satisfied by an $\mathcal{R}$-system $\mathcal{S}$ if there exists a natural transformation

$$
\alpha: t_{\mathcal{S}} \Rightarrow t_{\mathcal{S}}^{\prime}
$$

between the functors $t_{\mathcal{S}}, t_{\mathcal{S}}^{\prime}: \mathcal{S}^{n} \rightarrow \mathcal{S}$. We use the notation

$$
\mathcal{S} \models\left[t\left(x_{1}, \ldots, t_{n}\right)\right] \rightarrow\left[t^{\prime}\left(x_{1}, \ldots, x_{n}\right)\right] .
$$

With respect to this definition of satisfaction, the proof calculus is sound and complete (Meseguer 1992). Completeness is obtained by means of an initial model construction.

\subsection{The models of CRWL}

Before defining models we review some definitions. A partially ordered set (in short, poset) with bottom $\perp$ is a set $S$ equipped with a partial order $\sqsubseteq$ and a least element $\perp$. We say that an element $x \in S$ is totally defined if $x$ is maximal with respect

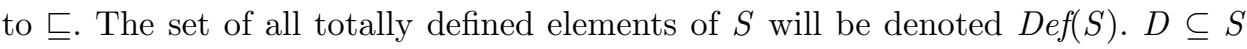
is a directed set if for all $x, y \in D$ there exists $z \in D$ with $x \sqsubseteq z, y \sqsubseteq z$. A subset $A \subseteq S$ is a cone if $\perp \in A$ and, for all $x \in A$ and $y \in S$, if $y \sqsubseteq x$ then $y \in A$. An ideal $I \subseteq S$ is a directed cone. For $x \in S$, the principal ideal generated by $x$ is $\langle x\rangle=\{y \in S \mid y \sqsubseteq x\}$. We write $\mathcal{C}(S)$ for the set of cones of $S$.

Given a signature $\Sigma$, a $C R W L$-algebra over $\Sigma$ is a triple

$$
\mathcal{A}=\left(D^{\mathcal{A}},\left\{c^{\mathcal{A}}\right\}_{c \in C_{\Sigma}},\left\{f^{\mathcal{A}}\right\}_{f \in F_{\Sigma}}\right)
$$

where $D^{\mathcal{A}}$ is a poset with bottom, and $c^{\mathcal{A}}$ and $f^{\mathcal{A}}$ are monotone mappings from $\left(D^{\mathcal{A}}\right)^{n}$ to $\mathcal{C}\left(D^{\mathcal{A}}\right)$, with $n$ the corresponding arity. In addition, for $c \in C_{\Sigma}^{n}$ and for all $u_{1}, \ldots, u_{n} \in D^{\mathcal{A}}$, there exists a $v \in D^{\mathcal{A}}$ such that $c^{\mathcal{A}}\left(u_{1}, \ldots, u_{n}\right)=\langle v\rangle$. Moreover, $v \in \operatorname{Def}\left(D^{\mathcal{A}}\right)$ in case that all $u_{i} \in \operatorname{Def}\left(D^{\mathcal{A}}\right)$.

Note that any $h: S \rightarrow \mathcal{C}\left(S^{\prime}\right)$ can be extended to a function $\hat{h}: \mathcal{C}(S) \rightarrow \mathcal{C}\left(S^{\prime}\right)$ defined by $\hat{h}(x)=\bigcup_{x \in S} h(x)$. By abuse of notation, we will write $\hat{h}$ also as $h$.

A valuation over $\mathcal{A}$ is any mapping $\eta: \mathcal{X} \rightarrow D^{\mathcal{A}}$, and we say that $\eta$ is totally defined if $\eta(x) \in \operatorname{Def}\left(D^{\mathcal{A}}\right)$ for all $x \in \mathcal{X}$. The evaluation of an expression $e \in$ $\operatorname{Expr}_{\perp}(\Sigma, \mathcal{X})$ in $\mathcal{A}$ under $\eta$ yields $\llbracket e \rrbracket^{\mathcal{A}} \eta \in \mathcal{C}\left(D^{\mathcal{A}}\right)$, which is defined recursively as follows:

- $\llbracket \perp \rrbracket^{\mathcal{A}} \eta=\langle\perp \mathcal{A}\rangle$.

- $\llbracket x \rrbracket^{\mathcal{A}} \eta=\langle\eta(x)\rangle$, for $x \in \mathcal{X}$.

- $\llbracket h\left(e_{1}, \ldots, e_{n}\right) \rrbracket^{\mathcal{A}} \eta=h^{\mathcal{A}}\left(\llbracket e_{1} \rrbracket^{\mathcal{A}} \eta, \ldots, \llbracket e_{n} \rrbracket^{\mathcal{A}} \eta\right)$, for all $h \in C_{\Sigma}^{n} \cup F_{\Sigma}^{n}$. 
We are now prepared to define models. Let $\mathcal{A}$ be CRWL-algebra $\mathcal{A}$ :

- $\mathcal{A}$ satisfies a reduction statement $a \rightarrow b$ under a valuation $\eta,(\mathcal{A}, \eta) \models a \rightarrow b$, if $\llbracket a \rrbracket^{\mathcal{A}} \eta \supseteq \llbracket b \rrbracket^{\mathcal{A}} \eta$.

- $\mathcal{A}$ satisfies a joinability statement $a \bowtie b$ under $\eta,(\mathcal{A}, \eta) \models a \bowtie b$, if $\llbracket a \rrbracket^{\mathcal{A}} \eta \cap$ $\llbracket b \rrbracket^{\mathcal{A}} \eta \cap \operatorname{Def}\left(D^{\mathcal{A}}\right) \neq \emptyset$.

- $\mathcal{A}$ satisfies a rule $l \rightarrow r \Leftarrow C$ if every valuation $\eta$ such that $(\mathcal{A}, \eta) \models C$ verifies $(\mathcal{A}, \eta) \models l \rightarrow r$.

- $\mathcal{A}$ is a model of $\Gamma, \mathcal{A} \models \Gamma$ if $\mathcal{A}$ satisfies all the rules in $\Gamma$.

As mentioned in Section 2.5 the CRWL-calculus is partially sound and complete (González-Moreno et al. 1999) with respect to this notion of satisfaction:

- If $\varphi$ is a reduction or a joinability statement, $\Gamma \vdash_{\mathrm{CRWL}} \varphi$ implies that $(\mathcal{A}, \eta) \models$ $\varphi$, for every $\mathcal{A} \models \Gamma$ and every totally defined valuation $\eta$.

- If $\varphi$ is a joinability statement or a reduction statement in which the righthand expression is a partial term, the previous implication becomes an equivalence.

Finally, we can also define homomorphisms between CRWL-algebras. Let $\mathcal{A}, \mathcal{B}$ be two CRWL-algebras over a signature $\Sigma$. A $C R W L$-homomorphism $H: \mathcal{A} \rightarrow \mathcal{B}$ is a monotone function $H: D^{\mathcal{A}} \rightarrow \mathcal{C}\left(D^{\mathcal{B}}\right)$ which satisfies the following conditions:

1. $H$ is element-valued: for all $u \in D^{\mathcal{A}}$ there exists $v \in D^{\mathcal{B}}$ such that $H(u)=\langle v\rangle$.

2. $H$ is strict: $H\left(\perp_{\mathcal{A}}\right)=\left\langle\perp_{\mathcal{B}}\right\rangle$.

3. $H$ preserves constructors: for all $c \in C_{\Sigma}^{n}, u_{i} \in D^{\mathcal{A}}$, is $H\left(c^{\mathcal{A}}\left(u_{1}, \ldots, u_{n}\right)\right)=$ $c^{\mathcal{B}}\left(H\left(u_{1}\right), \ldots, H\left(u_{n}\right)\right)$.

4. $H$ loosely preserves defined functions: that is, for all $f \in F_{\Sigma}^{n}, u_{i} \in D^{\mathcal{A}}$, $H\left(f^{\mathcal{A}}\left(u_{1}, \ldots, u_{n}\right)\right) \subseteq f^{\mathcal{B}}\left(H\left(u_{1}\right), \ldots, H\left(u_{n}\right)\right)$.

CRWL-algebras as objects with CRWL-homomorphisms as arrows form a category.

\subsection{An institution for CRWL}

An institution for CRWL was first defined in Molina-Bravo (2000). This institution, however, was defined with the goal of providing a basis for the semantics of modules in CRWL, and restricts its attention to a class of particular term algebras. Since our goal is more general, we do not place such a limitation and define $\mathcal{I}_{\mathrm{CRWL}}=$ (Sign, sen, Mod, $\models$ ) as follows:

- Sign: the category of signatures with constructors and signature morphisms.

- sen : Sign $\rightarrow$ Set the functor assigning to each signature $\Sigma$ the set of all conditional rewrite rules over it, and to each signature morphism $\sigma$ its homomorphic extension to rewrite rules, with $\sigma(\perp)=\perp$.

- Mod : Sign $^{\text {op }} \rightarrow$ Cat the functor assigning to each signature the category of CRWL-algebras and homomorphisms over it, and to each $\sigma: \Sigma \rightarrow \Sigma^{\prime}$ the forgetful functor mapping $\mathcal{A}^{\prime} \in\left|\operatorname{Mod}\left(\Sigma^{\prime}\right)\right|$ to the CRWL-algebra $\mathcal{A}_{\sigma}^{\prime}$ with the same underlying poset and such that $h^{\mathcal{A}_{\sigma}^{\prime}}=\sigma(h)^{\mathcal{A}^{\prime}}$ for all $h \in \Sigma$, and which is the identity over homomorphisms.

- $\models$ the satisfaction relation in CRWL. 
Proposition 7

$\mathcal{I}_{\mathrm{CRWL}}$ is an institution.

Proof

It is not difficult to check that Sign is a category, and that sen and Mod are indeed functors. As for the satisfaction condition, let $\sigma: \Sigma \rightarrow \Sigma^{\prime}$ be a signature morphism, $\mathcal{A}^{\prime} \in\left|\operatorname{Mod}\left(\Sigma^{\prime}\right)\right|$, and $\varphi \in \operatorname{sen}(\Sigma)$; we have to prove that

$$
\mathcal{A}^{\prime} \models \sigma(\varphi) \Leftrightarrow \mathcal{A}_{\sigma}^{\prime} \models \varphi .
$$

It is easy to show, by structural induction on $e$, that

$$
\llbracket e \rrbracket^{\mathcal{A}_{\sigma}^{\prime}} \eta=\llbracket \sigma(e) \rrbracket^{\mathcal{A}^{\prime}} \eta
$$

for every $e \in \operatorname{Expr}_{\perp}(\Sigma, \mathcal{X})$ and valuation $\eta$ over $\mathcal{A}^{\prime}$. Let $\varphi=e \rightarrow e^{\prime}$ be a reduction statement. Then, for any valuation $\eta$,

$$
\begin{aligned}
\left(\mathcal{A}^{\prime}, \eta\right) \models \sigma(\varphi) & \Leftrightarrow \quad \llbracket \sigma\left(e^{\prime}\right) \rrbracket^{\mathcal{A}^{\prime}} \eta \subseteq \llbracket \sigma(e) \rrbracket^{\mathcal{A}^{\prime}} \eta \\
& \Leftrightarrow \quad \llbracket e^{\prime} \rrbracket^{\mathcal{A}_{\sigma}^{\prime}} \eta \subseteq \llbracket e \rrbracket^{\mathcal{A}_{\sigma}^{\prime}} \eta \quad \Leftrightarrow \quad\left(\mathcal{A}_{\sigma}^{\prime}, \eta\right) \models \varphi
\end{aligned}
$$

and analogously for $\varphi$ a joinability statement. Now, if $l \rightarrow r \Leftarrow C$ is a conditional rewrite rule, it follows that $\mathcal{A}_{\sigma}^{\prime} \models C \Leftrightarrow \mathcal{A}^{\prime} \models \sigma(C)$ and $\mathcal{A}_{\sigma}^{\prime} \models l \rightarrow r \Leftrightarrow \mathcal{A}^{\prime} \models \sigma(l \rightarrow$ $r)$, and thus the satisfaction condition is indeed verified.

It can be proved that the category $\operatorname{Mod}(T)$ has products for every CRWL-theory $T$; it is not complete, however, as in Section 3.6 it is shown that, in general, $\operatorname{Mod}(T)$ does not have equalizers. $\mathcal{I}_{\text {CRWL }}$ is also a semiexact institution (Palomino 2001).

\subsection{An institution for $R L$}

The task of assigning an institution to RL is harder than expected. The first and most natural idea is to define the category of signatures Sign as the category of equational theories and theory morphisms, and the functor sen to map any such theory to the set of conditional rewrite rules over it. Since there are also notions of model and satisfaction in RL, the desired institution seems to be at hand. However, when one tries to put together the various components of the institution, problems start to arise.

In the first place, the notion of satisfaction in RL is defined only for unconditional rewrite rules, so our first task must be to extend its definition so as to encompass the conditional ones. Taking the definition of $\mathcal{R}$-systems as a guide, we say that a conditional rewrite rule

$$
[t(\bar{x})] \rightarrow\left[t^{\prime}(\bar{x})\right] \text { if }\left[a_{1}(\bar{x})\right] \rightarrow\left[b_{1}(\bar{x})\right] \wedge \ldots \wedge\left[a_{m}(\bar{x})\right] \rightarrow\left[b_{m}(\bar{x})\right]
$$

is satisfied by an $\mathcal{R}$-system $\mathcal{S}$ if there exists a natural transformation

$$
\alpha: t_{\mathcal{S}} \circ J_{\mathcal{S}} \Rightarrow t_{\mathcal{S}}^{\prime} \circ J_{\mathcal{S}}
$$

where $J_{\mathcal{S}}: \operatorname{Subeq}\left(\left(a_{j \mathcal{S}}, b_{j \mathcal{S}}\right)_{1 \leq j \leq m}\right) \rightarrow \mathcal{S}^{n}$. (Alternatively, one could also think of defining satisfaction by

$$
\mathcal{S} \models[t] \rightarrow\left[t^{\prime}\right] \text { if }\left[a_{1}\right] \rightarrow\left[b_{1}\right] \wedge \ldots \wedge\left[a_{m}\right] \rightarrow\left[b_{m}\right]
$$


if

$$
\mathcal{S} \models\left[a_{i}\right] \rightarrow\left[b_{i}\right] \quad i=1, \ldots, m \quad \Longrightarrow \quad \mathcal{S} \models[t] \rightarrow\left[t^{\prime}\right] .
$$

This option looks natural, but it is too loose in the sense that it requires the consequent to hold only if the condition is true for all possible instances. Note that, in our definition, the subequalizer is playing the same role valuations have in the definition of satisfaction in CRWL.)

We can now prove the following proposition, that justifies the soundness and completeness of the extended RL-calculus presented in Section 2.4

\section{Proposition 8}

Let $\mathcal{R}$ be an RL-theory and $[t(\bar{x})] \rightarrow\left[t^{\prime}(\bar{x})\right]$ if $\left[a_{1}(\bar{x})\right] \rightarrow\left[b_{1}(\bar{x})\right] \wedge \ldots \wedge\left[a_{m}(\bar{x})\right] \rightarrow$ $\left[b_{m}(\bar{x})\right]$ a conditional rewrite rule; then, the following statements are equivalent:

1. $\mathcal{R} \models[t(\bar{x})] \rightarrow\left[t^{\prime}(\bar{x})\right]$ if $\left[a_{1}(\bar{x})\right] \rightarrow\left[b_{1}(\bar{x})\right] \wedge \ldots \wedge\left[a_{m}(\bar{x})\right] \rightarrow\left[b_{m}(\bar{x})\right] ;$

2. $\mathcal{R}(\bar{x}) \cup\left\{\left[a_{1}(\bar{x})\right] \rightarrow\left[b_{1}(\bar{x})\right], \ldots,\left[a_{m}(\bar{x})\right] \rightarrow\left[b_{m}(\bar{x})\right]\right\} \models[t(\bar{x})] \rightarrow\left[t^{\prime}(\bar{x})\right] ;$

3. $\mathcal{R}(\bar{x}) \cup\left\{\left[a_{1}(\bar{x})\right] \rightarrow\left[b_{1}(\bar{x})\right], \ldots,\left[a_{m}(\bar{x})\right] \rightarrow\left[b_{m}(\bar{x})\right]\right\} \vdash[t(\bar{x})] \rightarrow\left[t^{\prime}(\bar{x})\right]$.

\section{Proof}

Statements (2) and (3) are equivalent by the soundness and completeness of the RL-calculus (Meseguer 1992). We will now prove that (1) implies (2) and then, that (3) implies (1).

To see that (1) implies (2), let $\mathcal{S}$ be an $\mathcal{R}(\bar{x}) \cup\left\{\left[a_{1}(\bar{x})\right] \rightarrow\left[b_{1}(\bar{x})\right], \ldots,\left[a_{m}(\bar{x})\right] \rightarrow\right.$ $\left.\left[b_{m}(\bar{x})\right]\right\}$-system. There exist, therefore, natural transformations

$$
h_{j}: a_{j}(\bar{x})_{\mathcal{S}} \rightarrow b_{j}(\bar{x})_{\mathcal{S}}
$$

for $j=1, \ldots, m$. Since in this context, that is, over $\Sigma(\bar{x})$, both $t(\bar{x})$ and $t^{\prime}(\bar{x})$ (as well as all the $a_{j}(\bar{x})$ and $\left.b_{j}(\bar{x})\right)$ are ground terms, we only need to find a morphism $t(\bar{x})_{\mathcal{S}} \rightarrow t^{\prime}(\bar{x})_{\mathcal{S}}$ in $\mathcal{S}$ to prove that $\mathcal{S} \models[t(\bar{x})] \rightarrow\left[t^{\prime}(\bar{x})\right]$, and it turns out that each $h_{j}$ is just a single morphism. Let us write $\left.\mathcal{S}\right|_{\Sigma}$ for the restriction of $\mathcal{S}$ to the signature $\Sigma$ (that is, $\left.\mathcal{S}\right|_{\Sigma}$ is like $\mathcal{S}$ but we forget the interpretations for $\bar{x}$ ). Clearly, $\left.\mathcal{S}\right|_{\Sigma}$ is an $\mathcal{R}$-system and therefore, by hypothesis, there exists a natural transformation

$$
\alpha: t_{\left.\mathcal{S}\right|_{\Sigma}} \circ J_{\left.\mathcal{S}\right|_{\Sigma}} \Rightarrow t_{\left.\mathcal{S}\right|_{\Sigma}}^{\prime} \circ J_{\left.\mathcal{S}\right|_{\Sigma}}
$$

where $J_{\left.\mathcal{S}\right|_{\Sigma}}:\left.\operatorname{Subeq}\left(\left(a_{\left.j \mathcal{S}\right|_{\Sigma}}, b_{\left.j \mathcal{S}\right|_{\Sigma}}\right)_{1 \leq j \leq m}\right) \rightarrow \mathcal{S}\right|_{\Sigma}{ }^{n}$. Because of the $h_{j}, 1 \leq j \leq m$, and noting that $a_{j}(\bar{x})_{\mathcal{S}}=a_{\left.j \mathcal{S}\right|_{\Sigma}}\left(\bar{x}_{\mathcal{S}}\right)$ (and analogously for $b_{j}$ ), the interpretation $\bar{x}_{\mathcal{S}}$ of the variables $\bar{x}$ in $\mathcal{S}$ belongs to the subequalizer: $\left(\bar{x}_{\mathcal{S}}, \bar{h}\right) \in \operatorname{Subeq}\left(\left(a_{\left.j \mathcal{S}\right|_{\Sigma}}, b_{\left.j \mathcal{S}\right|_{\Sigma}}\right)_{1 \leq j \leq m}\right)$. But then $\alpha\left(\bar{x}_{\mathcal{S}}, \bar{h}\right)$ is a morphism $t_{\left.\mathcal{S}\right|_{\Sigma}}\left(\bar{x}_{\mathcal{S}}\right) \rightarrow t_{\left.\mathcal{S}\right|_{\Sigma}}^{\prime}\left(\bar{x}_{\mathcal{S}}\right)$ in $\left.\mathcal{S}\right|_{\Sigma}$, and therefore a morphism $t(\bar{x})_{\mathcal{S}} \rightarrow t^{\prime}(\bar{x})_{\mathcal{S}}$ in $\mathcal{S}$, as required.

To show that (3) implies (1), given an $\mathcal{R}$-system $\mathcal{S}$ we will prove by induction on the derivation that

$$
\mathcal{S} \models[t(\bar{x})] \rightarrow\left[t^{\prime}(\bar{x})\right] \text { if }\left[a_{1}(\bar{x})\right] \rightarrow\left[b_{1}(\bar{x})\right] \wedge \ldots \wedge\left[a_{m}(\bar{x})\right] \rightarrow\left[b_{m}(\bar{x})\right] .
$$

According to the last rule of deduction employed:

- Reflexivity. It must be $[t]=\left[t^{\prime}\right]$ and the result is immediate. 
- Congruence. If the last step in the derivation is

$$
\frac{\left[t_{1}\right] \rightarrow\left[t_{1}^{\prime}\right] \quad \ldots \quad\left[t_{p}\right] \rightarrow\left[t_{p}^{\prime}\right]}{\left[f\left(t_{1}, \ldots, t_{p}\right)\right] \rightarrow\left[f\left(t_{1}^{\prime}, \ldots, t_{p}^{\prime}\right)\right]}
$$

we have, by the induction hypothesis,

$$
\mathcal{S} \models\left[t_{i}\right] \rightarrow\left[t_{i}^{\prime}\right] \text { if }\left[a_{1}\right] \rightarrow\left[b_{1}\right] \wedge \ldots \wedge\left[a_{m}\right] \rightarrow\left[b_{m}\right] \quad 1 \leq i \leq p,
$$

and there exist natural transformations $\alpha_{i}: t_{i \mathcal{S}} \circ J_{\mathcal{S}} \Rightarrow t_{i \mathcal{S}}^{\prime} \circ J_{\mathcal{S}}, 1 \leq i \leq p$, where $J_{\mathcal{S}}: \operatorname{Subeq}\left(\left(a_{j \mathcal{S}}, b_{j \mathcal{S}}\right)_{1 \leq j \leq m}\right) \rightarrow \mathcal{S}^{n}$. Let $(\bar{s}, \bar{m}) \in \operatorname{Subeq}\left(\left(a_{j \mathcal{S}}, b_{j \mathcal{S}}\right)_{1 \leq j \leq m}\right)$; if we define

$$
\alpha(\bar{s}, \bar{m})=f_{\mathcal{S}}\left(\alpha_{1}(\bar{s}, \bar{m}), \ldots, \alpha_{p}(\bar{s}, \bar{m})\right),
$$

we obtain a natural transformation $\alpha: f\left(t_{1}, \ldots, t_{p}\right) \circ J_{\mathcal{S}} \Rightarrow f\left(t_{1}^{\prime}, \ldots, t_{n}^{\prime}\right) \circ J_{\mathcal{S}}$ and the result is proved. Some warning words are in order here. In the functor $J_{\mathcal{S}}$ : $\operatorname{Subeq}\left(\left(a_{j \mathcal{S}}, b_{j \mathcal{S}}\right)_{1 \leq j \leq m}\right) \rightarrow \mathcal{S}^{n}$, the $n$ appearing as superscript depends on the actual number of variables in the sentence $\left[t_{i}\right] \rightarrow\left[t_{i}^{\prime}\right]$ if $\left[a_{1}\right] \rightarrow\left[b_{1}\right] \wedge \ldots \wedge\left[a_{m}\right] \rightarrow\left[b_{m}\right]$ and, although the $a_{j}$ and $b_{j}$ are fixed, this is not the case for $t_{i}$ and $t_{i}^{\prime}$ and thus the $n$ may vary with each $i$. This would imply that the category $\operatorname{Subeq}\left(\left(a_{j \mathcal{S}}, b_{j \mathcal{S}}\right)_{1 \leq j \leq m}\right)$ could vary as well, since its objects are pairs whose first component is an object of $\mathcal{S}^{n}$, and then the definition of $\alpha$ given above would no longer be valid. However, this is only a technical nuisance because the extra variables that $t_{i}$ and $t_{i}^{\prime}$ may add are simply ignored by the functors $a_{j \mathcal{S}}$ and $b_{j \mathcal{S}}$, and everything could be made to fit properly by using projection functors that would preserve the natural transformations. This same remark applies to the remaining cases, too.

- Transitivity. If we have

$$
\frac{[t] \rightarrow\left[t^{\prime}\right] \quad\left[t^{\prime}\right] \rightarrow\left[t^{\prime \prime}\right]}{[t] \rightarrow\left[t^{\prime \prime}\right]}
$$

by induction hypothesis there exist natural transformations

$$
\alpha_{1}: t_{\mathcal{S}} \circ J_{\mathcal{S}} \Rightarrow t_{\mathcal{S}}^{\prime} \circ J_{\mathcal{S}} \quad \text { and } \quad \alpha_{2}: t_{\mathcal{S}}^{\prime} \circ J_{\mathcal{S}} \Rightarrow t_{\mathcal{S}}^{\prime \prime} \circ J_{\mathcal{S}}
$$

where $J_{\mathcal{S}}: \operatorname{Subeq}\left(\left(a_{j \mathcal{S}}, b_{j \mathcal{S}}\right)_{1 \leq j \leq m}\right) \rightarrow \mathcal{S}^{n}$; the composition $\alpha_{2} \circ \alpha_{1}$ gives the result.

- Replacement. We distinguish two cases:

1. The rule employed is one of the $\left[a_{j}(\bar{x})\right] \rightarrow\left[b_{j}(\bar{x})\right]$. Since the terms are ground we must have

$$
\overline{\left[a_{j}(\bar{x})\right] \rightarrow\left[b_{j}(\bar{x})\right]}
$$

But in this case, $\mathcal{S} \models\left[a_{i}\right] \rightarrow\left[b_{i}\right]$ if $\left[a_{1}\right] \rightarrow\left[b_{1}\right] \wedge \ldots \wedge\left[a_{m}\right] \rightarrow\left[b_{m}\right]$ follows because the construction of the subequalizer produces a natural transformation $\alpha_{j}: a_{j \mathcal{S}} \circ J_{\mathcal{S}} \Rightarrow b_{j \mathcal{S}} \circ J_{\mathcal{S}}$.

2. For some rule $[l(\bar{y})] \rightarrow[r(\bar{y})]$ if $\left[u_{1}(\bar{y})\right] \rightarrow\left[v_{1}(\bar{y})\right] \wedge \ldots \wedge\left[u_{q}(\bar{y})\right] \rightarrow\left[v_{q}(\bar{y})\right]$ in $\mathcal{R}$, we have

$$
\begin{aligned}
& {\left[w_{1}\right] \rightarrow\left[w_{1}^{\prime}\right] \quad \ldots \quad\left[w_{p}\right] \rightarrow\left[w_{p}^{\prime}\right]} \\
& \frac{\left[u_{1}(\bar{w} / \bar{y})\right] \rightarrow\left[v_{1}(\bar{w} / \bar{y})\right] \quad \ldots \quad\left[u_{q}(\bar{w} / \bar{y})\right] \rightarrow\left[v_{q}(\bar{w} / \bar{y})\right]}{[l(\bar{w} / \bar{y})] \rightarrow\left[r\left(\overline{w^{\prime}} / \bar{y}\right)\right]} .
\end{aligned}
$$


By the induction hypothesis there exist natural transformations

$$
\alpha_{i}: w_{i \mathcal{S}} \circ J_{\mathcal{S}} \Rightarrow w_{i \mathcal{S}}^{\prime} \circ J_{\mathcal{S}} \quad 1 \leq i \leq p,
$$

and

$$
\beta_{i}: u_{i}(\bar{w})_{\mathcal{S}} \circ J_{\mathcal{S}} \Rightarrow v_{i}(\bar{w})_{\mathcal{S}} \circ J_{\mathcal{S}} \quad 1 \leq i \leq q,
$$

where $J_{\mathcal{S}}$ : Subeq $\left(\left(a_{j \mathcal{S}}, b_{j \mathcal{S}}\right)_{1 \leq j \leq m}\right) \rightarrow \mathcal{S}^{n}$. Since $\mathcal{S}$ is an $\mathcal{R}$-system, there also exists a natural transformation

$$
\gamma: l_{\mathcal{S}} \circ J_{\mathcal{S}}^{\prime} \Rightarrow r_{\mathcal{S}} \circ J_{\mathcal{S}}^{\prime}
$$

where $J_{\mathcal{S}}^{\prime}$ : $\operatorname{Subeq}\left(\left(u_{j \mathcal{S}}, v_{j \mathcal{S}}\right)_{1 \leq j \leq q}\right) \rightarrow \mathcal{S}^{p}$. We now need to find a natural transformation $\alpha: l(\bar{w})_{\mathcal{S}} \circ J_{\mathcal{S}} \Rightarrow r\left(\overline{w^{\prime}}\right)_{\mathcal{S}} \circ J_{\mathcal{S}}$. For that, let $(\bar{s}, \bar{m})$ be an object in $\operatorname{Subeq}\left(\left(a_{j \mathcal{S}}, b_{j \mathcal{S}}\right)_{1 \leq j \leq m}\right)$; due to the morphisms $\beta_{i}(\bar{s}, \bar{m})$ it turns out that $\left.\overline{w_{\mathcal{S}}(\bar{s})}, \overline{\beta(\bar{s}, \bar{m})}\right)$ belongs to $\operatorname{Subeq}\left(\left(u_{j \mathcal{S}}, v_{j \mathcal{S}}\right)_{1 \leq j \leq q}\right)$ and we can define

$$
\alpha(\bar{s}, \bar{m})=r_{\mathcal{S}}(\overline{\alpha(\bar{s}, \bar{m})}) \circ \gamma\left(\overline{w_{\mathcal{S}}(\bar{s})}, \overline{\beta(\bar{s}, \bar{m})}\right),
$$

which finishes the proof.

A more serious problem, as far as the definition of an institution for RL is concerned, is posed by the functor Mod : $\mathbf{S i g n}^{\text {op }} \rightarrow$ Cat mapping signatures to models. The difficulty resides in the fact that, in RL, models are assigned directly to RL-theories instead of signatures, as it is customary in other logics. One obvious solution would be to consider a signature $(\Sigma, E)$ as a theory $\mathcal{R}=(\Sigma, E, \emptyset, \emptyset)$ with empty set of axioms (and labels), and to map $(\Sigma, E)$ to the category $\mathcal{R}$-Sys of models of $\mathcal{R}$. But this approach presents an important drawback. Up to this point in the paper, we have omitted any explicit mention of the set of labels of an RL-theory. Although this was a safe convention when talking about deduction, it is no longer the case when our interest shifts to models. Thanks to the set of labels $L$ in an RLtheory $\mathcal{R}=(\Sigma, E, L, \Gamma)$, the elements of $\Gamma$ become special, labeled rewrite rules. These rules force $\mathcal{R}$-systems to have a certain internal structure: not only must $\mathcal{R}$-systems satisfy them, but must also associate to them a distinguished interpretation (natural transformation) that must be preserved by homomorphisms. (In particular, the same rule could appear twice in an RL-theory $\mathcal{R}$ under two different labels. $\mathcal{R}$-systems are then forced to provide two, possibly different, interpretations for the same rule, each of them to be preserved by the homomorphisms.) When considering a signature as a theory with empty sets of axioms, we are not taking into account labeled rewrite rules. This way, homomorphisms are not subjected to preserve any rewrite rule and the categories $\operatorname{Mod}(\Gamma)$ of models of $\Gamma$ and $\mathcal{R}$-Sys of $\mathcal{R}$-systems, that we expected to be the same, turn out to be different.

In Palomino (2001), some others attempts at defining an institution with Sign as the category of equational theories are explored but, since they cannot reflect the distinction between labeled rules belonging to RL-theories and unlabeled rules, all of them are bound to failure. For this reason we are led to an institution in which the category Sign subsumes all the information of an RL-theory. More precisely, we define $\mathcal{I}_{\mathrm{RL}}=($ Sign, sen, $\mathbf{M o d}, \models)$ where: 
- Sign is the discrete category of RL-theories.

- sen : Sign $\rightarrow$ Set maps each RL-theory to the set of conditional rewrite rules that can be built over its signature.

- Mod : Sign $^{\text {op }} \rightarrow$ Cat maps an RL-theory $\mathcal{R}$ to the category $\mathcal{R}$-Sys.

- $\models$ the satisfaction relation conveniently extended to conditional rewrite rules as discussed above.

Since Sign is discrete, this trivially defines an institution. Admittedly, this restriction seems to be not justified. In fact, two types of morphisms of RL-theories are proposed in Meseguer (1990). Basically, they are morphisms of equational theories "preserving" the rules in the RL-theories. For our purposes, however, the present definition is general enough as it stands and its extension would not modify the use we will make of it in the next section.

There exist other institutions associated to (variants of) RL in the literature, e.g., Cengarle 1998 Diaconescu and Futatsugi 2002); in these papers, the objects in the category of signatures are the sets of function symbols, without any rules. As a consequence of this simplicity and the reasons we have mentioned above, the general categorical models of RL must be somehow restricted and the choice in these two works is to require them to be preorders instead of arbitrary categories.

\subsection{Searching for embeddings}

Now that we have institutions associated to both RL and CRWL capturing formally their semantics, we would like to relate them by means of maps of institutions having "nice properties." In particular, due to the generality of RL and its $\mathcal{R}$-systems, a

natural question to ask is whether $\mathcal{I}_{\mathrm{CRWL}}$ can be considered as a subinstitution of $\mathcal{I}_{\mathrm{RL}}$.

The formal definition of subinstitution appeared originally in Meseguer (1989) and has been further generalized in subsequent works. One of those extensions was introduced in Meseguer (1998), where it is called an embedding. Embeddings are very general: the only requirement they impose on a map of institutions $(\Phi, \alpha, \beta)$ : $\mathcal{I} \rightarrow \mathcal{I}^{\prime}$ is that for each $T \in\left|\mathbf{T h}_{\mathcal{I}}\right|$, the functor $\beta_{T}: \operatorname{Mod}^{\prime}(\Phi(T)) \rightarrow \operatorname{Mod}(T)$ has to be an equivalence of categories.

We will show, however, that there is no embedding from $\mathcal{I}_{\mathrm{CRWL}}$ into $\mathcal{I}_{\mathrm{RL}}$. For that, it will be enough to find a categorical property which is preserved by an equivalence of categories and a theory $T \in\left|\mathbf{T h}_{\mathrm{CRWL}}\right|$ such that $\operatorname{Mod}_{\mathrm{RL}}(\Phi(T))$ satisfies it whereas $\operatorname{Mod}_{\mathrm{CRWL}}(T)$ does not.

Let $\Sigma$ be a signature with constructors such that $C_{\Sigma}=\emptyset$ and $F_{\Sigma}$ consists of just two constants $f_{1}$ and $f_{2}, \Gamma=\left\{f_{2} \rightarrow x \Leftarrow f_{1} \bowtie f_{1}\right\}$, and consider the CRWLtheory $T=(\Sigma, \Gamma)$. We define two CRWL-algebras over $\Sigma: \mathcal{A}$ given by the set $D^{\mathcal{A}}=$ $\left\{\perp, a_{1}, a_{2}\right\}$ with partial order $\perp \sqsubseteq a_{1} \sqsubseteq a_{2}$, and the cones $f_{1}^{\mathcal{A}}=\left\langle a_{1}\right\rangle$ and $f_{2}^{\mathcal{A}}=\langle\perp\rangle$; and $\mathcal{B}$ with $D^{\mathcal{B}}=\left\{\perp, b_{1}\right\}$, and the cones $f_{1}^{\mathcal{B}}=f_{2}^{\mathcal{B}}=\langle\perp\rangle . \mathcal{A}, \mathcal{B} \in\left|\operatorname{Mod}_{\mathrm{CRWL}}(T)\right|$ trivially, because they do not satisfy the condition $f_{1} \bowtie f_{1}$.

Let us now define two CRWL-homomorphisms $F, G: \mathcal{A} \rightarrow \mathcal{B}$, given by:

$$
F(x)=\langle\perp\rangle \quad \text { and } \quad G(x)= \begin{cases}\langle\perp\rangle & \text { if } x=\perp, a_{1} \\ \left\langle b_{1}\right\rangle & \text { if } x=a_{2}\end{cases}
$$


Clearly, $F$ and $G$ preserve both $f_{1}$ and $f_{2}$, so that they are actually homomorphisms; we will prove that there is no equalizer of $F$ and $G$. For let us assume that $E: \mathcal{E} \rightarrow \mathcal{A}$ is such an equalizer and let $H: \mathcal{A} \rightarrow \mathcal{A}$ be the homomorphism given by

$$
H(x)= \begin{cases}\langle\perp\rangle & \text { if } x=\perp \\ \left\langle a_{1}\right\rangle & \text { if } x=a_{1}, a_{2},\end{cases}
$$

satisfying $F \circ H=G \circ H$. Then, there must exist a unique homomorphism $M$ : $\mathcal{A} \rightarrow \mathcal{E}$ such that $E \circ M=H$. Let $e_{1}$ be the element in $\mathcal{E}$ such that $M\left(a_{1}\right)=\left\langle e_{1}\right\rangle$ and $E\left(e_{1}\right)=\left\langle a_{1}\right\rangle$. Since $E$ and $M$ loosely preserve defined functions,

$$
E\left(f_{2}^{\mathcal{E}}\right) \subseteq f_{2}^{\mathcal{A}}=\langle\perp\rangle
$$

and hence $e_{1} \notin f_{2}^{\mathcal{E}}$, and $\left\langle e_{1}\right\rangle=M\left(f_{1}^{\mathcal{A}}\right) \subseteq f_{1}^{\mathcal{E}}$. Therefore, since $\mathcal{E} \in \operatorname{Mod}_{\mathrm{CRWL}}(T)$, there must exist $e_{2} \in \mathcal{E}$ such that $e_{1} \sqsubset e_{2}$ : otherwise, $\mathcal{E}$ would satisfy $f_{1} \bowtie f_{1}$ but, since $e_{1} \notin f_{2}^{\mathcal{E}}$, not $\Gamma$. Besides, due to the monotonicity of $E$ and the equality $F \circ E=G \circ E$, it is $E\left(e_{2}\right)=\left\langle a_{1}\right\rangle$. But then we have $M_{1}, M_{2}: \mathcal{B} \rightarrow \mathcal{E}$ given by

$$
M_{1}(x)=\left\{\begin{array}{ll}
\langle\perp\rangle & \text { if } x=\perp \\
\left\langle e_{1}\right\rangle & \text { if } x=b_{1}
\end{array} \quad \text { and } \quad M_{2}(x)= \begin{cases}\langle\perp\rangle & \text { if } x=\perp \\
\left\langle e_{2}\right\rangle & \text { if } x=b_{1},\end{cases}\right.
$$

two different homomorphisms satisfying $E \circ M_{1}=E \circ M_{2}$, a contradiction with the universal property of equalizers.

In contrast with what happens in CRWL, the following proposition shows a construction for equalizers in RL.

\section{Proposition 9}

For all RL-theories $\mathcal{R}=(\Sigma, E, L, \Gamma)$, the category $\mathcal{R}$-Sys has equalizers.

\section{Proof}

Let $\mathcal{S}_{1}$ and $\mathcal{S}_{2}$ be two $\mathcal{R}$-systems and let $F, G: \mathcal{S}_{1} \rightarrow \mathcal{S}_{2}$ be two $\mathcal{R}$-homomorphisms between them; let us build their equalizer $E: \mathcal{E} \rightarrow \mathcal{S}_{1}$.

The objects in the category $\mathcal{E}$ are those $s \in \mathcal{S}_{1}$ such that $F(s)=G(s)$; the arrows, those $f: s \rightarrow s^{\prime}$ in $\mathcal{S}_{1}$ such that $F(f)=G(f)$ (which implies, in particular, that $F$ and $G$ also coincide over $s$ and $s^{\prime}$ ); composition is that of $\mathcal{S}_{1} \cdot \mathcal{E}$ is well-defined because functors preserve identities and composition.

Next, we assign a $(\Sigma, E)$-algebra structure to $\mathcal{E}$. For each $f \in \Sigma$ of arity $n$ we define $f_{\mathcal{E}}$ to be $\left.f_{\mathcal{S}_{1}}\right|_{\mathcal{E}}$, the restriction of $f_{\mathcal{S}_{1}}$ to $\mathcal{E}$. Let us check that this is a valid definition. If $e_{1}, \ldots, e_{n} \in|\mathcal{E}|$ then

$$
\begin{aligned}
F\left(f_{\mathcal{S}_{1}}\left(e_{1}, \ldots, e_{n}\right)\right) & =f_{\mathcal{S}_{2}}\left(F\left(e_{1}\right), \ldots, F\left(e_{n}\right)\right) & & (F \text { is homomorphism }) \\
& =f_{\mathcal{S}_{2}}\left(G\left(e_{1}\right), \ldots, G\left(e_{n}\right)\right) & & \left(e_{i} \in|\mathcal{E}|\right) \\
& =G\left(f_{\mathcal{S}_{1}}\left(e_{1}, \ldots, e_{n}\right)\right) & & (G \text { is homomorphism })
\end{aligned}
$$

and thus $f_{\mathcal{S}_{1}}\left(e_{1}, \ldots, e_{n}\right) \in|\mathcal{E}|$. Analogously for arrows. With this definition it is easy to prove by structural induction that $t_{\mathcal{E}}=\left.t_{\mathcal{S}_{1}}\right|_{\mathcal{E}^{n}}$ for all $t\left(x_{1}, \ldots, x_{n}\right) \in T_{\Sigma}(\mathcal{X})$. Therefore, for each $t=t^{\prime} \in E$ it is $t_{\mathcal{E}}=t_{\mathcal{E}}^{\prime}$.

The only thing missing in the definition of $\mathcal{E}$ are the natural transformations associated to the rewrite rules. Let

$$
r:[t(\bar{x})] \rightarrow\left[t^{\prime}(\bar{x})\right] \text { if }\left[a_{1}(\bar{x})\right] \rightarrow\left[b_{1}(\bar{x})\right] \wedge \ldots \wedge\left[a_{m}(\bar{x})\right] \rightarrow\left[b_{m}(\bar{x})\right]
$$


be a rule in $\mathcal{R}$. We have to define a natural transformation

$$
r_{\mathcal{E}}: t_{\mathcal{E}} \circ J_{\mathcal{E}} \Rightarrow t_{\mathcal{E}}^{\prime} \circ J_{\mathcal{E}}
$$

where $J_{\mathcal{E}}: \operatorname{Subeq}\left(\left(a_{j \mathcal{E}}, b_{j \mathcal{E}}\right)_{1 \leq j \leq m}\right) \rightarrow \mathcal{E}^{n}$ is the subequalizer functor. Using the construction of Section 3.2 and the fact that $a_{j \mathcal{E}}=\left.a_{j \mathcal{S}_{1}}\right|_{\mathcal{E}^{n}}$ and $b_{j \mathcal{E}}=\left.b_{j \mathcal{S}_{1}}\right|_{\mathcal{E}^{n}}, 1 \leq j \leq$ $m$, it follows that $\operatorname{Subeq}\left(\left(a_{j \mathcal{E}}, b_{j \mathcal{E}}\right)_{1 \leq j \leq m}\right)$ is a subcategory of $\operatorname{Subeq}\left(\left(a_{j \mathcal{S}_{1}}, b_{j} \mathcal{S}_{1}\right)_{1 \leq j \leq m}\right)$ and that $J_{\mathcal{E}}$ is just the restriction of the corresponding $J_{\mathcal{S}_{1}}$. Then we can define $r_{\mathcal{E}}$ simply by restricting $r_{\mathcal{S}_{1}}$, which is obviously a natural transformation, and this finishes our construction of $\mathcal{E}$ as an $\mathcal{R}$-system.

Let us now move to the definition of $E$ and the proof that it is an $\mathcal{R}$-homomorphism. $E$ is simply the inclusion functor. If $f \in \Sigma$ and $e_{1}, \ldots, e_{n} \in|\mathcal{E}|$, then

$$
E\left(f_{\mathcal{E}}\left(e_{1}, \ldots, e_{n}\right)\right)=f_{\mathcal{E}}\left(e_{1}, \ldots, e_{n}\right)=f_{\mathcal{E}}\left(E\left(e_{1}\right), \ldots, E\left(e_{n}\right)\right),
$$

so $E$ is a $\Sigma$-algebra homomorphism.

For a rewrite rule $r:[t(\bar{x})] \rightarrow\left[t^{\prime}(\bar{x})\right]$ if $\left[a_{1}(\bar{x})\right] \rightarrow\left[b_{1}(\bar{x})\right] \wedge \ldots \wedge\left[a_{m}(\bar{x})\right] \rightarrow\left[b_{m}(\bar{x})\right]$ in $\Gamma$, we have to show that the natural transformation $E \circ r_{\mathcal{E}}$ is equal to $r_{\mathcal{S}_{1}} \circ E^{\bullet}$. Let $\left(\bar{e}^{n}, \bar{u}^{m}\right) \in \operatorname{Subeq}\left(\left(a_{j \mathcal{E}}, b_{j \mathcal{E}}\right)_{1 \leq j \leq m}\right)$. Regarding $E^{\bullet}$, we only need to know that $E^{\bullet}\left(\bar{e}^{n}, \bar{u}^{m}\right)=\left(E^{n}\left(\bar{e}^{n}\right), E^{m}\left(\bar{u}^{m}\right)\right)$. Now,

$$
\begin{aligned}
& \left(E \circ r_{\mathcal{E}}\right)\left(\bar{e}^{n}, \bar{u}^{m}\right)=E\left(r_{\mathcal{E}}\left(\bar{e}^{n}, \bar{u}^{m}\right)\right)=r_{\mathcal{E}}\left(\bar{e}^{n}, \bar{u}^{m}\right) \\
& =r_{\mathcal{S}_{1}}\left(\bar{e}^{n}, \bar{u}^{m}\right)=r_{\mathcal{S}_{1}}\left(E^{n}\left(\bar{e}^{n}\right), E^{m}\left(\bar{u}^{m}\right)\right) \\
& =r_{\mathcal{S}_{1}}\left(E^{\bullet}\left(\bar{e}^{n}, \bar{u}^{m}\right)\right)=\left(r_{\mathcal{S}_{1}} \circ E^{\bullet}\right)\left(\bar{e}^{n}, \bar{u}^{m}\right) \text {, }
\end{aligned}
$$

so $E$ is an $\mathcal{R}$-homomorphism.

We already know that $\mathcal{E}$ is an $\mathcal{R}$-system and that $E$ is an $\mathcal{R}$-homomorphism; the only missing thing is the equalizer property. Let then $H: \mathcal{C} \rightarrow \mathcal{S}_{1}$ be an $\mathcal{R}$ homomorphism such that $F \circ H=G \circ H$; we have to find a unique $M: \mathcal{C} \rightarrow \mathcal{E}$ such that $E \circ M=H$. As $E$ is the inclusion functor the uniqueness is clear, because the only possibility for all objects $c$ and arrows $u$ in $\mathcal{C}$ is $M(c)=H(c)$ and $M(u)=$ $H(u)$. It remains to prove that this is a valid definition. First, because of the equality $F \circ H=G \circ H$ the image of $H$ is included in $\mathcal{E}$ and $M$ is well-defined; as $H$ is a functor, so is $M$. Given $f \in \Sigma$ and $c_{1}, \ldots, c_{n} \in|\mathcal{C}|$, we have

$$
\begin{aligned}
M\left(f_{\mathcal{C}}\left(c_{1}, \ldots, c_{n}\right)\right) & =H\left(f_{\mathcal{C}}\left(c_{1}, \ldots, c_{n}\right)\right) \\
& =f_{\mathcal{S}_{1}}\left(H\left(c_{1}\right), \ldots, H\left(c_{n}\right)\right) \\
& =f_{\mathcal{E}}\left(M\left(c_{1}\right), \ldots, M\left(c_{n}\right)\right)
\end{aligned}
$$

and $M$ is a $\Sigma$-algebra homomorphism. Finally, if $r:[t(\bar{x})] \rightarrow\left[t^{\prime}(\bar{x})\right]$ if $\left[a_{1}(\bar{x})\right] \rightarrow$ $\left[b_{1}(\bar{x})\right] \wedge \ldots \wedge\left[a_{m}(\bar{x})\right] \rightarrow\left[b_{m}(\bar{x})\right]$ is a rewrite rule in $\Gamma$ and $\left(\bar{c}^{n}, \bar{u}^{m}\right)$ is an object of Subeq $\left(\left(a_{j \mathcal{C}}, b_{j \mathcal{C}}\right)_{1 \leq j \leq m}\right)$, then

$$
\begin{array}{rlrl}
r_{\mathcal{E}}\left(M^{\bullet}\left(\bar{c}^{n}, \bar{u}^{m}\right)\right) & =r_{\mathcal{E}}\left(M^{n}\left(\bar{c}^{n}\right), M^{m}\left(\bar{u}^{m}\right)\right) & =r_{\mathcal{S}_{1}}\left(H^{n}\left(\bar{c}^{n}\right), H^{m}\left(\bar{u}^{m}\right)\right) \\
& =r_{\mathcal{S}_{1}}\left(H^{\bullet}\left(\bar{c}^{n}, \bar{u}^{m}\right)\right) & & =H\left(r_{\mathcal{C}}\left(\bar{c}^{n}, \bar{u}^{m}\right)\right) \\
& =M\left(r_{\mathcal{C}}\left(\bar{c}^{n}, \bar{u}^{m}\right)\right) & &
\end{array}
$$

and we have $M \circ r_{\mathcal{C}}=r_{\mathcal{E}} \circ M^{\bullet}$.

Note that, in the above proof, the equalizer $\mathcal{E}$ is a model of all the rewrite rules that $\mathcal{S}_{1}$ satisfies. Therefore the result is still valid when $\mathcal{R}$-Sys is replaced by the category $\operatorname{Mod}_{\mathrm{RL}}(\Gamma)$ for some set $\Gamma$ of rewrite rules in the institution $\mathcal{I}_{\mathrm{RL}}$. Since an equivalence of categories preserves limits, we have: 
Proposition 10

$\mathcal{I}_{\mathrm{CRWL}}$ is not embeddable in $\mathcal{I}_{\mathrm{RL}}$.

Proof

Let $T$ be the CRWL-theory defined at the beginning of this section. It has been shown that $\operatorname{Mod}_{\mathrm{CRWL}}(T)$ does not have all equalizers, whereas $\operatorname{Mod}_{\mathrm{RL}}(\Phi(T))$ has, regardless of the actual definition of $\Phi$. Therefore, there cannot exist an equivalence of categories $\beta_{T}: \operatorname{Mod}_{\mathrm{RL}}(\Phi(T)) \rightarrow \operatorname{Mod}_{\mathrm{CRWL}}(\Phi)$.

What about the other way around? Can we embed $\mathcal{I}_{\mathrm{RL}}$ in $\mathcal{I}_{\mathrm{CRWL}}$ ? When we began preparing this work our intuition was that we would be able to view CRWL as a "sublogic" of RL in the first place, but also that the converse would not be true. The previous discussion has shown that our intuition was wrong about the first point and our goal now is to deal with the second.

In order to prove that RL cannot be embedded in CRWL we have to find an RL-theory $T$ such that $\operatorname{Mod}_{\mathrm{RL}}(T)$ has a categorical property that no category of models in CRWL has. In order to do that, note that for any CRWL-theory $T$ there exists a CRWL-algebra $\mathcal{A} \in\left|\operatorname{Mod}_{\mathrm{CRWL}}(T)\right|$ with an infinite number of automorphisms. Simply consider $\mathcal{A}$ given by $D^{\mathcal{A}}=\left\{\perp, a, b_{1}, b_{2}, \ldots\right\}$ with $\perp \sqsubseteq a$, $\perp \sqsubseteq b_{1} \sqsubseteq b_{2} \sqsubseteq \ldots$. the image of all functions associated to constructor symbols to be $\langle a\rangle$, and the corresponding one for all defined function symbols to be $D^{\mathcal{A}}$. This way $\mathcal{A}$ is clearly a CRWL-algebra, satisfies all conditional rewrite rules, and the set $\left\{F_{i}: \mathcal{A} \rightarrow \mathcal{A}\right\}_{i \in \mathbb{N}}$, where

$$
F_{i}(x)= \begin{cases}\langle\perp\rangle & \text { if } x=\perp \\ \langle a\rangle & \text { if } x=a \\ \left\langle b_{i}\right\rangle & \text { if } x=b_{j}(j \in \mathbb{N})\end{cases}
$$

is an infinite family of automorphisms of $\mathcal{A}$. On the other hand, in RL, if $\mathcal{R}$ is the RL-theory given by $(\{c\},\{x=c\}, \emptyset, \emptyset)$ then, for all $\mathcal{R}$-systems $\mathcal{S}$, the equality $i d_{\mathcal{S}}=c_{\mathcal{S}}$, where $c_{\mathcal{S}}$ is a constant functor, forces $\mathcal{S}$ to be a category with just one object and one arrow, and no infinite family of homomorphisms can exist. Therefore (as an equivalence of categories is full and faithful), $\operatorname{Mod}_{\mathrm{RL}}(\mathcal{R})$ is not categorically equivalent to $\operatorname{Mod}_{\mathrm{CRWL}}(\Phi(\mathcal{R}))$, whatever $\Phi$ might be, and we have:

Proposition 11

$\mathcal{I}_{\mathrm{RL}}$ is not embeddable in $\mathcal{I}_{\mathrm{CRWL}}$.

Let us note that Propositions 10 and 11] still hold even if the general semantics of RL is replaced by the preorder semantics mentioned on page 25 On the other hand, maps of institutions could be given for the trivial semantics in which either everything or nothing can be proven in both logics.

\section{Conclusions}

The main outcome of the research carried out in this paper has been the clarification of the relationship between RL and CRWL. Both logics have been proved to be expressive enough to simulate deduction in each other in a simple way, though 
resorting to binary predicates. On the other hand, the results on institutions have shown that neither can RL be considered as a sublogic of CRWL, nor can CRWL with respect to RL.

During the preparation of this work we have been forced to take a close look at the notions of entailment system and institution, and the difficulties we have found have shown us that intuition can be misleading in this field. The conclusion we have reached is that it would be very convenient to develop some kind of generalization of these concepts. One reason supporting this claim is the fact that, although it seems clear that CRWL should fit within the framework of entailment systems, the lack of the transitivity property forbids it to be considered so. In addition, there have been several occasions wherein we have had to make a distinction between two types of sentences within the same logic. The most outstanding case was that of labeled and unlabeled rewrite rules in RL, but we should also emphasize that rules in CRWL-theories are a restricted class of the more general class of reduction statements. What all these examples have in common is that sentences belonging to a theory are given a different treatment from the rest of sentences and, with the current definitions of entailment system and institution, there is no way of taking this distinction into account.

Finally, though not presented in the paper due to lack of space, the results in Section 2 can be used to show that CRWL is reflective (Palomino 2001). Intuitively, this property means that the logic can reason about itself and has been fruitfully exploited in RL in the design of programs; thus, an interesting open line of research consists in the study of ways by which reflection can be exploited in CRWL.

\section{Acknowledgments}

The author warmly thanks Narciso Martí-Oliet, Mario Rodríguez-Artalejo, and José Meseguer for their help in the preparation of this work.

\section{References}

Arenas-Sánchez, P. And Rodríguez-Artalejo, M. 2001. A general framework for lazy functional logic programming with algebraic polymorphic types. Theory and Practice of Logic Programming 1, 2, 185-245.

Barr, M. And Wells, C. 1999. Category Theory for Computing Science. Third Edition. Centre de Recherches Mathématiques.

Bosco, P. G., Giovannetti, E., And Moiso, C. 1988. Narrowing vs. SLD-resolution. Theoretical Computer Science 59, 3-23.

Cengarle, M. V. 1998. The rewriting logic institution. Tech. Rep. 9801, LudwigMaximilians-Universität München, Institut für Informatik. May.

Diaconescu, R. And Futatsugi, K. 2002. Logical foundations of CafeOBJ. Theoretical Computer Science 285, 2, 289-318.

Goguen, J. And Burstall, R. 1992. Institutions: Abstract model theory for specification and programming. Journal of the Association for Computing Machinery 39, 1, 95-146.

Goguen, J. And Roşu, G. 2002. Institution morphisms. Formal Aspects of Computing $13,3-5,274-307$. 
González-Moreno, J. C., Hortalá-GonzÁlez, M. T., López-Fraguas, F. J., And Rodríguez-Artalejo, M. 1999. An approach to declarative programming based on a rewriting logic. Journal of Logic Programming 40, 47-87.

GonzÁlez-Moreno, J. C., Hortalá-GonzÁlez, M. T., and Rodríguez-Artalejo, M. 2001. Polymorphic types in functional logic programming. Journal of Functional and Logic Programming 2001, 1. Special Issue 1, http://danae.uni-muenster.de/lehre/kuchen/JFLP

LAmbeK, J. 1970. Subequalizers. Canadian Mathematical Bulletin 13, 337-349.

Martí-Oliet, N. And Meseguer, J. 2002a. Rewriting logic as a logical and semantic framework. In Handbook of Philosophical Logic. Second Edition, D. Gabbay, Ed. Vol. 9. Kluwer Academic Press, 1-81.

Martí-Oliet, N. And Meseguer, J. 2002b. Rewriting logic: Roadmap and bibliography. Theoretical Computer Science 285, 2, 121-154.

Meseguer, J. 1989. General logics. In Logic Colloquium'87, H.-D. Ebbinghaus, J. Fernández-Prida, M. Garrido, D. Lascar, and M. Rodríguez-Artalejo, Eds. NorthHolland, 275-329.

Meseguer, J. 1990. Rewriting as a unified model of concurrency. Tech. Rep. SRI-CSL90-02, SRI International, Computer Science Laboratory. Feb. Revised June 1990.

Meseguer, J. 1992. Conditional rewriting logic as a unified model of concurrency. Theoretical Computer Science 96, 1, 73-155.

Meseguer, J. 1998. Membership algebra as a logical framework for equational specification. In Recent Trends in Algebraic Development Techniques, 12th International Workshop, WADT'97, Tarquinia, Italy, June 3 - 7, 1997, Selected Papers, F. ParisiPresicce, Ed. Lecture Notes in Computer Science, vol. 1376. Springer-Verlag, 18-61.

Meseguer, J. 2000. Rewriting logic and Maude: Concepts and applications. In Rewriting Techniques and Applications, 11th International Conference, RTA 2000, Norwich, UK, July 10-12, 2000, Proceedings, L. Bachmair, Ed. Lecture Notes in Computer Science, vol. 1833. Springer-Verlag, 1-26.

Mryoshi, H. 1996. Modelling conditional rewriting logic in structured categories. In Proceedings First International Workshop on Rewriting Logic and its Applications, WRLA'96, Asilomar, California, September 3-6, 1996, J. Meseguer, Ed. Electronic Notes in Theoretical Computer Science, vol. 4. Elsevier, 20-34. http://www.elsevier.com/locate/entcs/volume4.html

Molina-Bravo, J. M. 2000. Modularidad en programación lógico-funcional de primer orden. Ph.D. thesis, Universidad de Málaga, Spain.

PAlomino, M. 2001. Relating Meseguer's rewriting logic and the constructor-based rewriting logic. M.S. thesis, Facultad de Matemáticas, Universidad Complutense de Madrid. http://maude.cs.uiuc.edu/papers

Thati, P., Sen, K., and Martí-Oliet, N. 2002. An executable specification of asynchronous pi-calculus. In Proceedings Fourth International Workshop on Rewriting Logic and its Applications, WRLA'02, Pisa, Italy, September 19-21, 2002, F. Gadducci and U. Montanari, Eds. Electronic Notes in Theoretical Computer Science, vol. 71. Elsevier.

Verdejo, A. 2003. Técnicas de especificación formal de sistemas orientados a objetos basadas en lógica de reescritura. Ph.D. thesis, Universidad Complutense de Madrid, Spain.

Verdejo, A. And Martí-Oliet, N. 2002. Implementing CCS in Maude2. In Proceedings Fourth International Workshop on Rewriting Logic and its Applications, WRLA'02, Pisa, Italy, September 19-21, 2002, F. Gadducci and U. Montanari, Eds. Electronic Notes in Theoretical Computer Science, vol. 71. Elsevier. 


\section{Appendix A Proofs}

Proposition 1

$\mathcal{E}_{\mathrm{RL}}=($ Sign, sen,$\vdash)$ is an entailment system.

Proof

The fact that composition of signature morphisms is associative (for equational logics in general, and for our unsorted and unconditional case in particular) is all that is needed to check that Sign is a category and sen a functor. Regarding the properties that $\vdash$ must satisfy:

1. reflexivity: By Replacement (combined with Implication introduction for conditional rules).

2. monotonicity: Immediate by the definition of the entailment relation.

3. transitivity: Assume $\Gamma \vdash \varphi_{i}$ for all $i \in I$ and $\Gamma \cup\left\{\varphi_{i} \mid i \in I\right\} \vdash \psi$. The easiest way to prove $\Gamma \vdash \psi$ is by resorting to the soundness and completeness of the RL-calculus. Let $\mathcal{S}$ be a $\Gamma$-system, so $\mathcal{S} \models \varphi_{i}$ for all $i \in I$. Therefore $\Gamma$ can also be considered a $\Gamma \cup\left\{\varphi_{i} \mid i \in I\right\}$-system and then $\mathcal{S} \models \psi$.

4. $\vdash$-translation: Suppose $\Gamma \vdash \varphi$. Given a theory morphism $H$, it can be proved by induction on the derivation that $\operatorname{sen}(H)(\Gamma) \vdash \operatorname{sen}(H)(\varphi)$. The only nontrivial case is the one corresponding to Replacement and we illustrate it with an unconditional rule. If, for some $[t(\bar{x})] \rightarrow\left[t^{\prime}(\bar{x})\right] \in \Gamma$, the last step in the derivation of $\Gamma \vdash \varphi$ is

$$
\frac{\left[w_{1}\right] \rightarrow\left[w_{1}^{\prime}\right] \quad \ldots \quad\left[w_{n}\right] \rightarrow\left[w_{n}^{\prime}\right]}{[t(\bar{w} / \bar{x})] \rightarrow\left[t^{\prime}\left(\overline{w^{\prime}} / \bar{x}\right)\right]}
$$

then, by the induction hypothesis, $\operatorname{sen}(H)(\Gamma) \vdash \operatorname{sen}(H)\left(\left[w_{i}\right] \rightarrow\left[w_{i}^{\prime}\right]\right)$ for $i=$ $1, \ldots, n$, and, since $\operatorname{sen}(H)\left([t] \rightarrow\left[t^{\prime}\right]\right)=[H(t)] \rightarrow\left[H\left(t^{\prime}\right)\right]$ belongs to $\operatorname{sen}(H)(\Gamma)$, we can build a derivation for $\operatorname{sen}(H)\left([t(\bar{w} / \bar{x})] \rightarrow\left[t^{\prime}\left(\overline{w^{\prime}} / \bar{x}\right)\right]\right)$ from $\operatorname{sen}(H)(\Gamma)$ by applying Replacement.

Proposition 6

Given any RL-theory $T=(\Sigma, E, \Gamma)$, and $l, r \in T_{\Sigma}(\mathcal{X})$ :

$$
\begin{aligned}
T \vdash_{\mathrm{RL}}[l] \rightarrow[r] & \Leftrightarrow\left(\exists l^{\prime} \in[l], \exists r^{\prime} \in[r]\right) \quad \beta(T) \vdash_{\mathrm{CRWL}} R\left(l^{\prime}, r^{\prime}\right) \rightarrow \text { true } \\
& \Leftrightarrow\left(\forall l^{\prime} \in[l], \forall r^{\prime} \in[r]\right) \beta(T) \vdash_{\mathrm{CRWL}} R\left(l^{\prime}, r^{\prime}\right) \rightarrow \text { true }
\end{aligned}
$$

Proof

By Propositions 4 and $\left[5\right.$ it is enough to see that if $(\Sigma \cup\{t r u e\}, E, \Gamma) \vdash_{\mathrm{RL}}[l] \rightarrow[r]$ then $(\Sigma, E, \Gamma) \vdash_{\mathrm{RL}}[l] \rightarrow[r]$. The easiest way of proving this implication is by using the completeness of the RL-calculus.

Note that, since true does not belong to $\Sigma$ (and hence it appears neither in $E$ nor in $\Gamma$ ), a model of $(\Sigma \cup\{t r u e\}, E, \Gamma)$ is just a model of $(\Sigma, E, \Gamma)$ together with an interpretation for the constant true, and therefore either both satisfy $[l] \rightarrow[r]$ or none does. But then

$$
\begin{aligned}
(\Sigma, E, \Gamma) \vdash[l] \rightarrow[r] & \Leftrightarrow(\Sigma, E, \Gamma) \models[l] \rightarrow[r] \\
& \Leftrightarrow(\Sigma \cup\{\text { true }\}, E, \Gamma) \models[l] \rightarrow[r] \\
& \Leftrightarrow \quad(\Sigma \cup\{\text { true }\}, E, \Gamma) \vdash[l] \rightarrow[r]
\end{aligned}
$$

whence $(\Sigma, E, \Gamma) \vdash[l] \rightarrow[r]$ follows. 